\title{
SCATTERING METRICS AND GEODESIC FLOW AT INFINITY
}

\author{
RICHARD MELROSE AND MACIEJ ZWORSKI
}

\begin{abstract}
Any compact $\mathcal{C}^{\infty}$ manifold with boundary admits a Riemann metric on its interior taking the form $x^{-4} d x^{2}+x^{-2} h^{\prime}$ near the boundary, where $x$ is a boundary defining function and $h^{\prime}$ is a smooth symmetric 2-cotensor restricting to be positive-definite, and hence a metric, $h$, on the boundary. The scattering theory associated to the Laplacian for such a 'scattering metric' was discussed by the first author and here it is shown, as conjectured, that the scattering matrix is a Fourier integral operator which quantizes the geodesic flow on the boundary, for the metric $h$, at time $\pi$. To prove this the Poisson operator, of the associated generalized boundary problem, is constructed as a Fourier integral operator associated to a singular Legendre manifold.
\end{abstract}

\section{INTRODUCTION}

In this paper the geometric structure of the scattering matrix on certain asymptotically Euclidean spaces is considered. We show that the scattering matrix is a Fourier integral operator which quantizes the geodesic flow on the boundary (which is metrically at infinity) at time $\pi$.

The notion of an asymptotically Euclidean manifold is formulated in terms of a class of Riemannian metrics on the interiors of compact manifolds with boundary. This class, called scattering metrics, was introduced in [10] where the spectral, and scattering, theory for the corresponding Laplace operators was examined. As defined there, a scattering metric is a Riemannian metric on the interior, $X^{\circ}$, of the compact manifold with boundary, $X$, which can be brought to the form

$$
g=\frac{d x^{2}}{x^{4}}+\frac{h^{\prime}}{x^{2}}
$$

near the boundary. Here, $x \in \mathcal{C}^{\infty}(X)$ is a defining function for the boundary and $h^{\prime}$ is a smooth symmetric 2-cotensor which restricts to the boundary to a Riemann metric, $h$. These scattering metrics are generalizations of the Euclidean metric on $\mathbb{R}^{n}$ and the associated Laplacian, acting on functions or forms, has spectral and scattering theoretic properties similar to those of the flat Laplacian. Indeed one of the purposes of the discussion in [10] was to give a systematic development of scattering theory without relying on the symmetries of Euclidean space.

If $f \in \mathcal{C}^{\infty}(\partial X)$ is chosen then, as is shown in [10], for each $0 \neq \lambda \in \mathbb{R}$ there is a unique function $u \in \mathcal{C}^{\infty}\left(X^{\circ}\right)$ which satisfies $\left(\Delta-\lambda^{2}\right) u=0$ and is of the form

$$
u=e^{i \lambda / x} x^{\frac{n-1}{2}} f^{\prime}+e^{-i \lambda / x} x^{\frac{n-1}{2}} f^{\prime \prime}
$$

where $f^{\prime}, f^{\prime \prime} \in \mathcal{C}^{\infty}(X)$ and $\left.f^{\prime}\right|_{\partial X}=f$. The map

$$
A(\lambda):\left.\mathcal{C}^{\infty}(\partial X) \ni f \longmapsto f^{\prime \prime}\right|_{\partial X} \in \mathcal{C}^{\infty}(\partial X)
$$


is the (absolute) scattering matrix, at frequency $\lambda$. The convention here regarding signs is slightly different to that used in [10] (rather is that of [11]) so that the scattering matrix $A(\lambda)$ is the scattering matrix at $-\lambda$ in the convention of [10]. In this article we show:

Main theorem - The scattering matrix, $A(\lambda)$, of a scattering metric is, for $0 \neq$ $\lambda \in \mathbb{R}$, a Fourier integral operator on $\partial X$, of order 0 , associated to the canonical diffeomorphism

$$
\exp \left(\pi H_{\sqrt{h}}\right): T^{*} \partial X \backslash 0 \longrightarrow T^{*} \partial X \backslash 0
$$

given by geodesic flow at distance $\pi$ for the induced metric on $\partial X$.

The proof of the existence of generalized eigenfunctions of the form (0.2) given in [10] is essentially non-constructive. To analyse the microlocal structure of $A(\lambda)$ we need to proceed much more constructively. To do so we consider the behaviour of geodesics near the boundary, for a metric (0.1), and thereby construct a rescaled Legendre submanifold at infinity with which the generalized plane wave eigenfunctions for the Laplacian are associated as Lagrangian distributions, although in a somewhat different sense from the usual context of singularities. These generalized plane waves give, after normalization, the Schwartz kernel of the Poisson operator, which in this setting is the map

$$
P(\lambda): \mathcal{C}^{\infty}(\partial X) \ni f \longmapsto u \in \mathcal{C}^{\infty}\left(X^{\circ}\right)
$$

where $u$ is the eigenfunction in (0.2). The scattering matrix is, in an appropriate sense, a boundary value of $P(\lambda)$.

The basic example is given by the Euclidean case. Under the form of stereographic projection

$$
\begin{gathered}
\mathrm{SP}: \mathbb{R}^{n} \ni z \longmapsto\left(\left(1+|z|^{2}\right)^{-\frac{1}{2}}, z\left(1+|z|^{2}\right)^{-\frac{1}{2}}\right) \in \mathbb{S}_{+}^{n}, \\
\mathbb{S}_{+}^{n}=\left\{t=\left(t_{0}, \cdots, t_{n}\right) \in \mathbb{R}^{n+1} ; t_{0} \geq 0,|t|=1\right\},
\end{gathered}
$$

$\mathbb{R}^{n}$ is identified with $\left(\mathbb{S}_{+}^{n}\right)^{\circ}$ where $X=\mathbb{S}_{+}^{n}$ is the compact manifold with boundary. The Euclidean metric becomes

$$
|d z|^{2}=d r^{2}+r^{2}|d \omega|^{2}=\frac{d x^{2}}{x^{4}}+\frac{|d \omega|^{2}}{x^{2}},|z|=r=\frac{1}{x}, \omega=\frac{z}{|z|},
$$

where $|d \omega|^{2}$ is the standard metric on the sphere $\mathbb{S}^{n-1}=\partial \mathbb{S}_{+}^{n}$. This is precisely a scattering metric of the form (0.1). To compute the absolute scattering matrix for Euclidean space we follow the argument of Appendix to [8]; see also [11]. Thus consider $u(x, \omega)=\exp (i \lambda \omega \cdot \theta / x), \theta \in \mathbb{S}^{n-1}$. Then $\left(\Delta-\lambda^{2}\right) u=0$ and, in the sense of distributions in $\omega$, as $x \rightarrow 0$

$$
u(x, \omega)=e^{i \lambda \frac{\omega \cdot \theta}{x}} \sim(2 \pi)^{\frac{n-1}{2}}\left(\frac{x}{\lambda}\right)^{\frac{n-1}{2}}\left[e^{\frac{i \lambda}{x}} e^{-\frac{i \pi}{4}(n-1)} \delta_{\theta}(\omega)+e^{-\frac{i \lambda}{x}} e^{\frac{i \pi}{4}(n-1)} \delta_{-\theta}(\omega)\right]
$$

which follows from the stationary phase lemma applied in the variable $\omega$ after integration against a function in $\mathcal{C}^{\infty}\left(\mathbb{S}^{n-1}\right)$. Applying the definitions $(0.2)$ and $(0.3)$ we conclude that

$$
\begin{gathered}
A(\lambda): \mathcal{C}^{\infty}\left(\mathbb{S}^{n-1}\right) \ni f \longmapsto i^{n-1} j^{*} f \in \mathcal{C}^{\infty}\left(\mathbb{S}^{n-1}\right) \\
\text { where } j: \mathbb{S}^{n-1} \ni \omega \longmapsto-\omega \in \mathbb{S}^{n-1} .
\end{gathered}
$$


From the microlocal point of view the pull back by the antipodal map is a quantization of the geodesic flow on the sphere at time $\pi$.

As in the Euclidean case the addition of a short-range perturbation does not change the geometric structure of the scattering matrix in $\lambda \neq 0$ although it may add a finite number of $L^{2}$ eigenvalues to the spectrum. In the present setting of scattering metrics this can be generalized as follows. If $V \in x^{2} \mathcal{C}^{\infty}(X)$ then the theorem above remains valid for the scattering matrix for the operator $\Delta+V$; even the symbol of the scattering matrix remains unchanged. Let us also note the perturbations of Euclidean space which are covered by our analysis (although this is a very special case indeed). Suppose that $\tilde{g}$ is a smooth metric on Euclidean space which takes the form

$$
\tilde{g}_{i j}=\delta_{i j}+|z|^{-2} h_{i j}\left(\frac{z}{|z|}, \frac{1}{|z|}\right) \text { as }|z| \rightarrow \infty
$$

where the coefficients $h_{i j}$ are smooth on $\mathbb{S}^{n-1} \times[0,1)$. Then, under stereographic compactification (0.5), of $\mathbb{R}^{n}$ to $\mathbb{S}_{+}^{n}, \tilde{g}$ defines a scattering metric on $\mathbb{S}_{+}^{n}$. Again this is a short range condition on the perturbation in (0.9). For a recent discussion of scattering theory for metric perturbations of Euclidean space see [12]. Our construction of the Poisson operator has much in common with earlier work on scattering for long-range potentials; see in particular [1].

In $\S 1$ we outline our construction of the Poisson operator in a simple case, where the boundary is flat and there are no conjugate points for the geodesic flow, on the boundary, up to time $\pi$. The basic components of 'scattering geometry' are described in $\S 2$ and this is used to analyse the geodesic flow near infinity in $\S 3$. The rescaled Lagrangian, here identified as a Legendre manifold, with which the Poisson operator is associated is introduced in $\S 4$ and the parametrization of such Legendre manifolds is discussed in $\S 6$. The extra complications which arise from the presence of conic singularities are explained in $\S 7$. The simplest classes of Legendre distributions, namely oscillating functions, are described in $\S 8$. In $\S 9$ the Maslov bundle for Legendre manifolds is defined. The symbol mapping for the ring of scattering pseudodifferential operators on a general compact manifold with boundary is analysed in $\S 10$. We also note there that the semi-classical frequency set can be considered as a special case of the scattering wave front set defined in [10]. The general spaces of Legendre distributions and their symbolic properties are examined in $\S 11$ and $\S 12$, for a smooth Legendre manifold and for the case of manifolds with conic points in $\S 13$. A push-forward theorem for Legendre functions is proved in $\S 14$. These ingredients are combined in $\S 15$ where a parametrix for the Poisson operator is constructed as a Legendre distribution in this sense. This leads directly to the proof of the main theorem in $\S 16$, we also observe that the symbol of the scattering matrix is constant as a function of $\lambda>0$ and we conjecture that the trace formula for perturbations of Euclidean space, which expresses the regularized trace of the wave group in terms of the scattering phase (see for example [11] and [13]), should extend to this setting.

As already noted the construction of the parametrix for the Poisson operator uses a theory of Legendre functions associated to a Legendre manifold over the boundary. These can be viewed, locally, as Fourier transforms of the Lagrangian distributions introduced by Hörmander. We allow Legendre manifolds with certain conic singularities, so in this sense we also generalize the notion of a Lagrangian 
distribution. We make heavy use of the results and techniques of [5]. This paper also relies on the main results of [10].

\section{An EXAMPLE}

To orient the reader we begin by outlining our analysis of the scattering matrix for a simple example and then briefly discuss the modifications required to handle the general case. Let $\mathbb{T}^{n-1}=\left(\mathbb{S}^{1}\right)^{n-1}$ be a large torus, with flat metric, where by 'large' we mean that each point has a neighbourhood isometric to a ball in $\mathbb{R}^{n-1}$ of radius larger than $\pi$. This just means that each of the circles has radius greater than $\pi$. Our example is then

$$
X=\mathbb{D} \times \mathbb{T}^{n-2}
$$

where $\mathbb{D} \subset \mathbb{R}^{2}$ is the closed disk. On $X$ we take a metric of 'product type' near the boundary

$$
g=\frac{d x^{2}}{x^{4}}+\frac{|d y|^{2}}{x^{2}} \text { near } x=0
$$

where $x \in \mathcal{C}^{\infty}(\mathbb{D})$ is a boundary defining function and $|d y|^{2}$ in the flat metric on $\mathbb{T}^{n-1}$. The flatness of the boundary and the lack of conjugate points for the geodesic flow, on the torus, up to time $\pi$ make this easy to analyse. In fact we can do almost all the analysis on the non-compact, but convenient, model $X_{\mathrm{nc}}=(0, \infty)_{x} \times \mathbb{R}_{y}^{n-1}$ with metric (1.2), and then pass to $X$ at the last stage.

As already noted, to examine the microlocal structure of the scattering matrix we construct the Poisson operator, $P(\lambda)$, defined by $(0.4)$ and then obtain the scattering matrix as the outgoing boundary value of $P(\lambda)$ in the sense of $(0.3)$. Constructing $P(\lambda)$ reduces to constructing 'generalized plane wave solutions' which have $\delta$-functions as their boundary values. In the Euclidean case this is $(0.7)$.

For the model $X_{\mathrm{nc}}$ the Laplacian is

$$
\Delta=\left(x^{2} D_{x}\right)^{2}+i x(n-1) x^{2} D_{x}+x^{2}\left|D_{y}\right|^{2}
$$

and it is the same near the boundary for $X$. This operator is homogeneous of degree 2 under $x \longrightarrow s x, s>0$, so it suffices to consider the special cases $\lambda= \pm 1$. Complex conjugation reduces the case $\lambda=-1$ to $\lambda=1$, so we consider only this value of the frequency. Since the operator is also translation-invariant in $y$ we only need consider delta data supported at 0 . Thus, we wish to find a generalized plane wave solution satisfying

$$
\begin{aligned}
(\Delta-1) u & =0 \text { and } \\
\lim _{x \rightarrow 0} x^{-\frac{n-1}{2}} e^{-\frac{i}{x}} \int u(x, y) \phi(y) d y & =\phi(0) \forall \phi \in \mathcal{C}_{c}^{\infty}(\{|y|<\pi\}) .
\end{aligned}
$$

The 'ansatz' motivated by the Euclidean case is

$$
u_{1}(x, y)=e^{i \Phi(y) / x} a(x, y)
$$

where $\Phi$ satisfies the eikonal equation

$$
\Phi^{2}+\left|\partial_{y} \Phi\right|^{2}-1=0, \Phi(y) \sim 1-c|y|^{2}, y \sim 0, c \neq 0 .
$$

The solution to (1.6) is $\Phi(y)=\cos |y|$, so $c=\frac{1}{2}$ in (1.6). As we will see in $\S 6$ this is a particular example of the general notion of the parametrization of a scattering Legendrian submanifold. The non-degeneracy in this initial condition for $\Phi$ allows, using the principle of stationary phase, the second part of (1.4) to be arranged for 
$u_{1}$ of the form (1.5) and some amplitude $a$, at least when applied to $\phi$ supported close to 0 .

Consider the Taylor series at the boundary, $a(x, y)=a_{0}(y)+x a_{1}(y)+\cdots$, of the amplitude in (1.5). Applying $\Delta-1$ to $u_{1}$ gives transport equations for the coefficients $a_{j}$. The first of these, the coefficient of $x$, takes the form

$$
\frac{2}{i} L_{0} a_{0}=\frac{2}{i}\left(\partial_{y} \Phi \cdot \partial_{y}+\frac{n-1}{2} \Phi(y)+\frac{1}{2}\left|D_{y}\right|^{2} \Phi\right) a_{0}=0 .
$$

The vector field here is invariant under rotations in $y$ and, in terms of $r=|y|, L_{0}$ reduces to $r g\left(r^{2}\right) \frac{d}{d r}+r^{2} f\left(r^{2}\right)$ with $f$ and $g$ smooth and $g(0)=-1$. Thus, near $0,(1.7)$ has a unique solution satisfying $a_{0}=1+|y|^{2} h\left(|y|^{2}\right)$ with $h$ smooth. The higher transport equation are of the form

$$
L_{j} a_{j}=e_{j}, L_{j}=L_{0}-j \Phi \text { with } e_{j} \text { smooth. }
$$

They therefore have unique smooth solutions near 0 .

The solutions to (1.7) and (1.8) can be continued smoothly until the vector field degenerates again, which happens precisely at $|y|=\pi$, since then $\partial_{y} \Phi=0$. Summing the Taylor series for $a$ using Borel's Lemma this gives a solution, modulo terms vanishing to infinite order with $x$ to (1.4) of the form (1.5).

To continue this solution up to, and beyond, the singularity of the transport equations at $|y|=\pi$ we need a different 'ansatz' in place of (1.5). A systematic discussion of this will be given below in terms of distributions associated to Legendre manifolds with conic singularities. Explicitly, near $|y|=\pi$ we look for $u_{1}$ as an integral

$$
u_{1}(x, y)=x^{-\frac{1}{2}} \int_{0}^{\infty} e^{i \Psi(y, s) / x} s^{p} b\left(\frac{x}{s}, y, s, x\right) d s, s \in \mathbb{R}
$$

where $b \in \mathcal{C}_{c}^{\infty}\left([0, \infty) \times \mathbb{R}^{n-1} \times[0, \infty) \times[0, \infty)\right)$ and $\Psi$ is given by

$$
\Psi(y, s)=-1+f(y) s-\frac{1}{2} s^{2} \text { where } \cos |y|=-1+\frac{1}{2} f(y)^{2} \text { as }|y| \sim \pi .
$$

Thus $f(y) \sim \pi-|y|$, is a smooth function near $|y|=\pi$. We note that $\Psi$ also satisfies an eikonal equation

$$
|\Psi|^{2}+\left|\partial_{y} \Psi\right|^{2}-1=0 \text { when } \partial_{s} \Psi=0 .
$$

As will be discussed below in $\S 7$, this phase function corresponds to a parametrization of a Legendre submanifold with conic points, here in the 'trivial' case of a Legendrian with boundary. The stationary phase lemma allows $u_{1}$, as already defined in $|y|<\pi$, of the form (1.5) to be written in the form (1.9) near $|y|=\pi-\epsilon$, $\epsilon>0$ small, with smooth amplitude $a^{\prime}$.

In writing down the transport equations we use the 'blown up' coordinates $s$ and $X=x / s$. Putting $\tilde{b}(X, y, s)=s^{p} b(X, y, s, X s)$ the condition on the amplitude so that $(\Delta-1) u_{1}=f_{1}$ with $f_{1}$ of the same form but with amplitude vanishing faster than any power of $X$ is

$$
\begin{gathered}
\left(L_{0}^{\prime}+s X L_{1}^{\prime}\right) \tilde{b}(X, y, s) \equiv 0 \quad \bmod X^{\infty} \\
\left.\left.\tilde{b}(X, y, s)\right|_{s=\epsilon} \equiv a^{\prime}(X, y, s)\right|_{s=\epsilon} \quad \bmod X^{\infty} .
\end{gathered}
$$

Here

$$
L_{0}^{\prime}=s \partial_{s}-\frac{n-2}{2}-s^{2} W
$$


with $W$ and $L_{1}^{\prime}$ respectively first and second order differential operators in $\partial_{y}$, $s \partial_{s}$ and $X \partial_{X}$, and the initial condition $a^{\prime}$ comes from matching with the solution for $|y|<\pi$ obtained using (1.5). By writing $\tilde{b}$ and $a^{\prime}$ in power series in $X$ with coefficients depending on $y$ and $s,(1.13)$ can be solved iteratively. The constant term $(n-2) / 2$ determines the structure of the solution and in fact $\tilde{b}=s^{(n-2) / 2} b$ with

$$
\left(L_{0}^{\prime}+s X L_{1}^{\prime}\right) s^{(n-2) / 2} b(X, y, s) \equiv 0 \bmod s^{n / 2} X^{\infty} .
$$

Thus $p=\frac{1}{2}(n-2)$ in $(1.9)$.

These arguments show the existence of $u_{1}$ of the form (1.5) for $|y|<\pi$ and (1.9) for $|y| \sim \pi$, satisfying the incoming boundary condition in (1.4) (the incoming data is the coefficient of $\exp (i / x))$ near 0 and $(\Delta-1) u_{1}=f_{1}$, where $\chi f_{1}=\mathcal{O}\left(x^{\infty}\right)$ for $\chi \in \mathcal{C}^{\infty}(X)$ supported away from $|y|=\pi$. Near $|y|=\pi$

$$
f_{1}(x, y)=x^{-\frac{1}{2}} \int_{0}^{\infty} e^{i \Psi(y, s) / x} x s^{\frac{n}{2}} \alpha\left(\frac{x}{s}, y, s\right) d s,
$$

where $\alpha \in X^{\infty} \mathcal{C}_{c}^{\infty}\left([0, \infty) \times \mathbb{R}^{n-1} \times[0, \infty)\right)$. From this it follows that

$$
f_{1} \in x^{(n+3) / 2} e^{-\frac{i}{x}} \mathcal{C}^{\infty}\left([0, \infty) \times \mathbb{R}^{n-1}\right) .
$$

So far, this construction has all been on the non-compact model $X_{\mathrm{nc}}$. However, $u_{1}$ can be taken to have support in $|y|<\pi+\epsilon$ and $x<\epsilon$ for any $\epsilon>0$. Choosing $\epsilon>0$ small enough this allows $u_{1}$ to be transferred to the compact manifold $X$ in (1.1). Then, by an iteration argument (see $\S 12$ of [10] and Lemma 16 below), $u_{1}$ can be perturbed to an exact eigenfunction by adding a term of the form

$$
v_{1} \in x^{(n-1) / 2} \exp (-i / x) \mathcal{C}^{\infty}(X)
$$

such that $(\Delta-1) v_{1}=-f_{1}$.

Hence the solution to (1.4) is given by $u=u_{1}+v_{1}$ and to study the singular part of the outgoing boundary data of $u$ (the coefficient of $\exp (-i / x)$ ) we only need to consider the $u_{1}$ term. Since $\Phi$ in (1.5) is non-degenerate for $0<|y|<\pi$, the outgoing boundary value comes from the term of the form (1.9). For $\phi \in \mathcal{C}_{c}^{\infty}\left(\mathbb{R}^{n-1}\right)$ supported near $|y|=\pi$, using (1.10)

$$
\begin{aligned}
\lim _{x \rightarrow 0} e^{\frac{i}{x}} x^{-\frac{n-1}{2}} & \int_{\mathbb{R}^{n-1}} u_{1}(x, y) \phi(y) d y \\
& =\lim _{x \rightarrow 0} e^{\frac{i}{x}} x^{-\frac{n-1}{2}} x^{-\frac{1}{2}} \int_{\mathbb{R}^{n-1}} \int_{0}^{\infty} e^{i\left(-1+f(y) s-\frac{1}{2} s^{2}\right) / x} s^{\frac{n-2}{2}} b\left(\frac{x}{s}, y, s\right) \phi(y) d s d y \\
& =\lim _{x \rightarrow 0} \int_{\mathbb{R}^{n-1}} \int_{0}^{\infty} e^{i f(y) \eta-i \frac{1}{2} x \eta^{2}} \eta^{\frac{n-2}{2}} b\left(\frac{1}{\eta}, y, x \eta\right) \phi(y) d \eta d y \\
& =\int_{\mathbb{R}^{n-1}} \int_{0}^{\infty} e^{i f(y) \eta} \eta^{\frac{n-2}{2}} b\left(\frac{1}{\eta}, y, 0\right) \phi(y) d \eta d y \\
& =\left\langle T\left(\delta_{0}\right), \phi\right\rangle,
\end{aligned}
$$

where $T \in I^{0}\left(\mathbb{R}^{n-1} \times \mathbb{R}^{n-1}, \mathcal{G}_{\pi}^{\prime}\right)$ is a Fourier integral operator of order zero associated to the canonical transformation

$$
\mathcal{G}_{\pi}:(y, \eta) \longmapsto(y+\pi \eta /|\eta|, \eta) .
$$


In fact, with a different amplitude, its kernel is given, up to a smooth term, by

$$
\int e^{i(|x-y|-\pi) \eta} \eta^{\frac{n-2}{2}} b^{\sharp}\left(\frac{1}{\eta}, x-y, 0\right) d \eta .
$$

This demonstrates the theorem in this simple case.

In the general case of a compact manifold with scattering metric we proceed to construct the generalized plane wave by very much the same method. Indeed the construction near the 'initial' point is essentially the same, except that it is more convenient to construct all the plane waves at once, i.e. to work on $X \times \partial X$ rather than $X$ with a selected boundary point. The transport equation is then related to geodesic flow on the boundary and so will in general have conjugate points before parameter time $\pi$. We therefore need to replace the simple ansatz (1.5) by a superposition of such functions. To do so we develop the theory of such 'Legendre' distributions in close analogy with Hörmander's theory of Lagrangian distributions. We also develop a similar theory of superpositions of functions of the form (1.9) which are associated to Legendre manifolds with conic points.

\section{SCATTERING BUNDLE}

Let $X$ be a compact manifold with boundary. A metric of the form (0.1) is associated to a rescaled tangent bundle on $X$. Namely, the space of smooth vector fields on $X$ of bounded length with respect to the metric is the Lie algebra

$$
\begin{aligned}
\mathcal{V}_{\mathrm{sc}}(X)=x \mathcal{V}_{\mathrm{b}}(X)=\left\{V \in \mathcal{C}^{\infty}(X ; T X) ; V=x W,\right. \\
\\
\left.W \in \mathcal{C}^{\infty}(X ; T X) \text { is tangent to the boundary }\right\} .
\end{aligned}
$$

As discussed in [10], $\S 2$, there is a natural vector bundle ${ }^{\mathrm{sc}} T X$, over $X$, such that $\mathcal{V}_{\mathrm{sc}}(X)=\mathcal{C}^{\infty}\left(X ;{ }^{\mathrm{sc}} T X\right)$. Near a boundary point ${ }^{\mathrm{sc}} T X$ is spanned by $x^{2} \partial_{x}$ and the $x \partial_{y}$ where $x$ is a local boundary defining function and $y_{1}, \ldots, y_{n-1}$ are tangential coordinates. Restriction to the interior extends to define a smooth bundle map $\iota:{ }^{\mathrm{sc}} T X \longrightarrow T X$ which is an isomorphism over the interior of $X$ and vanishes identically over $\partial X$.

A scattering metric, (0.1), defines a non-degenerate fibre metric on ${ }^{\mathrm{sc}} T X$. If ${ }^{\mathrm{sc}} T^{*} X$ is the dual bundle to ${ }^{\mathrm{sc}} T X$ then the metric determines, and is determined by, the square of the length $g \in \mathcal{C}^{\infty}\left({ }^{\mathrm{sc}} T^{*} X\right)$. Since this function is also the joint symbol (see [10] and $\S 10$ below) of the Laplacian

$$
j(\Delta)=g
$$

we are especially interested in the behaviour of its associated Hamiltonian vector field, that is, in the rescaled symplectic geometry of ${ }^{\mathrm{sc}} T^{*} X$. To give a uniform discussion of this rescaling near the boundary of $X$ and the usual rescaling near infinity on the fibres of the cotangent bundle we consider the compactified scattering cotangent bundle ${ }^{\mathrm{sc}} \bar{T}^{*} X$ as our basic 'microlocal' space. This is the compact manifold with corners obtained by stereographic compactification of the fibres of ${ }^{\mathrm{sc}} T^{*} X$.

As a compact manifold with corners $Y={ }^{\mathrm{sc}} \bar{T}^{*} X$ has a natural 'scattering structure' analogous to (2.1) and reducing to it away from the corner. Namely, if 
Figure 1. The 'microlocal' space ${ }^{\mathrm{sc}} \bar{T}^{*} X$.

$\rho \in \mathcal{C}^{\infty}(Y)$ is the product of boundary defining functions for the boundary hypersurfaces of $Y$ (a 'total boundary defining function') then

$$
\begin{aligned}
\mathcal{V}_{\mathrm{sc}}(Y)=\rho \mathcal{V}_{\mathrm{b}}(Y)= & \left\{V \in \mathcal{C}^{\infty}(Y ; T Y) ; V=\rho W\right. \text { where } \\
& \left.W \in \mathcal{C}^{\infty}(Y ; T Y) \text { is tangent to all boundary faces }\right\} .
\end{aligned}
$$

Here $\rho=\rho_{\sigma} \rho_{\partial}$ where $\left\{\rho_{\partial}=0\right\}={ }^{\mathrm{sc}} T_{\partial X}^{*} X$ is the part of the bundle over the boundary and $\left\{\rho_{\sigma}=0\right\}={ }^{\mathrm{sc}} S^{*} X$ is the boundary added to compactify ${ }^{\mathrm{sc}} T^{*} X$. Again $\mathcal{V}_{\mathrm{sc}}(Y)=\mathcal{C}^{\infty}\left(Y ;{ }^{\mathrm{sc}} T Y\right)$ for a natural vector bundle over $Y$. The reason for introducing (2.3) is its relationship to the contact structure.

Lemma 1. The canonical 1-form on $T^{*} X$ extends from the interior to a smooth section of the bundle ${ }^{s c} T^{*}\left({ }^{s c} \bar{T}^{*} X\right)$, that is, ${ }^{s c} T^{*} Y$ for $Y={ }^{s c} \bar{T}^{*} X$.

Proof. Let $x \in \mathcal{C}^{\infty}(X)$ be a local boundary defining function and let $y_{1}, \ldots, y_{n-1}$ be additional coordinates near a boundary point. The tautological 1-form is

$$
\alpha=\xi d x+\eta \cdot d y
$$

where $\xi, \eta_{1}, \ldots, \eta_{n-1}$ are the canonically dual coordinates in the fibres of $T^{*} X$. Now $d x / x^{2}$ and $d y_{j} / x$, for $j=1, \ldots, n-1$ give a local basis for ${ }^{\mathrm{sc}} T^{*} X$. The induced canonical coordinates on ${ }^{\mathrm{sc}} T^{*} X$ are then $x, y_{1}, \ldots, y_{n-1}, \tau$ and $\mu_{1}, \ldots, \mu_{n-1}$ where a general point of ${ }^{\mathrm{sc}} T^{*} X$ is

$$
\tau \frac{d x}{x^{2}}+\mu \cdot \frac{d y}{x} .
$$

In terms of these coordinates the map $\iota^{*}: T^{*} X \longrightarrow{ }^{\mathrm{sc}} T^{*} X$, dual to $\iota:{ }^{\mathrm{sc}} T X \longrightarrow$ $T X$, is $(x, \eta) \longmapsto\left(x^{2} \xi, x \eta\right)=(\tau, \mu)$. Thus the 1 -form $\alpha$ lifts to

$$
{ }^{\mathrm{sc}} \alpha=\tau \frac{d x}{x^{2}}+\mu \cdot \frac{d y}{x} \in \mathcal{C}^{\infty}\left({ }^{\mathrm{sc}} T^{*} X ;{ }^{\mathrm{sc}} T^{*}\left({ }^{\mathrm{sc}} T^{*} X\right)\right) .
$$

This proves the stated smoothness of ${ }^{\mathrm{sc}} \alpha$ over the interior over $Y$ except near the sphere bundle 'at infinity.' 
Stereographic compactification of the fibres gives coordinates in ${ }^{\mathrm{sc}} \bar{T}^{*} X$

$$
Z=\frac{(1, \tau, \mu)}{\left(1+\tau^{2}+|\mu|^{2}\right)^{\frac{1}{2}}} \in \mathbb{S}_{+}^{n}=\left\{Z=\left(Z_{0}, Z^{\prime}\right) \in \mathbb{R}^{n+1} ; Z_{0} \geq 0,|Z|=1\right\}
$$

Since $Z_{0}=\left(1+\tau^{2}+|\mu|^{2}\right)^{-\frac{1}{2}}$ is a defining function for the boundary, (2.6) becomes

$$
{ }^{\mathrm{sc}} \alpha=Z_{1} \frac{d x}{Z_{0} x^{2}}+Z^{\prime} \cdot \frac{d y}{Z_{0} x} \in \mathcal{C}^{\infty}\left(Y ;{ }^{\mathrm{sc}} T^{*} Y\right), Y={ }^{\mathrm{sc}} \bar{T}^{*} X .
$$

Now,

$$
\frac{d x}{Z_{0} x^{2}}, \frac{d Z_{0}}{Z_{0}^{2} x}, \frac{d Z^{\prime}}{Z_{0} x}, \frac{d y}{Z_{0} x}
$$

is a basis for ${ }^{\mathrm{sc}} T^{*} Y$ so this proves the lemma.

Let ${ }^{\mathrm{sc}} \Lambda^{k} Y$ be the $k$-fold exterior power of ${ }^{\mathrm{sc}} T^{*} Y$, so in particular ${ }^{\mathrm{sc}} \Lambda^{1} Y={ }^{\mathrm{sc}} T^{*} Y$. The exterior derivative gives a map

$$
d: \mathcal{C}^{\infty}\left(Y ;{ }^{\mathrm{sc}} \Lambda^{k} Y\right) \longrightarrow \mathcal{C}^{\infty}\left(Y ;{ }^{\mathrm{sc}} \Lambda^{k+1} Y\right)
$$

However, for the canonical 1-form on $Y={ }^{\mathrm{sc}} \bar{T}^{*} X, d^{\mathrm{sc}} \alpha \in Z_{0} x \mathcal{C}^{\infty}\left(Y ;{ }^{\mathrm{sc}} \Lambda^{2} Y\right)$ since

$$
\begin{gathered}
d^{\mathrm{sc}} \alpha=d \tau \wedge \frac{d x}{x^{2}}+d \mu \wedge \frac{d y}{x}+\mu \cdot \frac{d y}{x} \wedge \frac{d x}{x} \\
=Z_{0} x \times\left(\frac{d Z_{1}}{x Z_{0}} \wedge \frac{d x}{x^{2} Z_{0}}-Z_{1} \frac{d Z_{0}}{Z_{0}^{2} x} \wedge \frac{d x}{Z_{0} x}+\frac{d Z^{\prime \prime}}{x Z_{0}} \wedge \frac{d y}{x Z_{0}}\right. \\
\left.-\frac{d Z_{0}}{Z_{0}^{2} x} \wedge Z^{\prime \prime} \cdot \frac{d y}{x Z_{0}}+Z^{\prime \prime} \cdot \frac{d u}{Z_{0} x} \wedge \frac{d x}{x^{2} Z_{0}}\right)
\end{gathered}
$$

where $Z^{\prime \prime}=\left(Z_{2}, \ldots, Z_{n}\right)$. Thus, as a scattering form, $d^{\text {sc }} \alpha$ vanishes at both boundaries of $Y={ }^{\mathrm{sc}} \bar{T}^{*} X$. Furthermore, as is clear from $(2.11), d^{\mathrm{sc}} \alpha / Z_{0} x$ is a nondegenerate smooth section of ${ }^{\mathrm{sc}} \Lambda^{2} Y$. Contraction with $d^{\mathrm{sc}} \alpha$ therefore defines an isomorphism

$$
\mathcal{V}_{\mathrm{b}}(Y)=\frac{1}{Z_{0} x} \mathcal{V}_{\mathrm{sc}}(Y) \longleftrightarrow \mathcal{C}^{\infty}\left(Y ;{ }^{\mathrm{sc}} \Lambda^{1}\right)
$$

In fact consider the space $\rho_{\sigma}^{-m} \rho_{\partial}^{k} \mathcal{C}^{\infty}(Y)$, where $\rho_{\sigma}$ and $\rho_{\partial}$ are defining functions for the two boundaries of $Y={ }^{\mathrm{sc}} \bar{T}^{*} X,\left\{\rho_{\sigma}=0\right\}$ being the sphere bundle of infinity. Exterior differentiation gives

$$
d: \rho_{\sigma}^{-m} \rho_{\partial}^{k} \mathcal{C}^{\infty}(Y) \longrightarrow \rho_{\sigma}^{-m+1} \rho_{\partial}^{k+1} \mathcal{C}^{\infty}\left(Y ;{ }^{\mathrm{sc}} \Lambda^{1} Y\right)
$$

and hence, composing with the inverse of (2.12), gives the Hamilton map

$$
\rho_{\sigma}^{-m} \rho_{\partial}^{k} \mathcal{C}^{\infty}(Y) \ni p \longmapsto{ }^{\mathrm{sc}} H_{p} \in \rho_{\sigma}^{-m+1} \rho_{\partial}^{k+1} \mathcal{V}_{\mathrm{b}}(Y)
$$

In view of the extra vanishing factors here we define

$$
{ }^{\mathrm{sc}} H_{p}^{m, k}=\left(\rho_{\sigma}^{m-1} \rho_{\partial}^{-k-1}\right){ }^{\mathrm{sc}} H_{p} \in \mathcal{V}_{\mathrm{b}}(Y) \text { for } p \in \rho_{\sigma}^{-m} \rho_{\partial}^{k} \mathcal{C}^{\infty}(Y)
$$

the rescaled scattering Hamilton vector field. Note that this definition depends on the choice of $\rho_{\sigma}$ and $\rho_{\partial}$. 
We refer the reader to [10] for a discussion of the more elementary constructions and properties of 'scattering geometry.' For example the scattering density bundle ${ }^{\mathrm{sc}} \Omega$ and more importantly the corresponding half-density bundle ${ }^{\mathrm{sc}} \Omega^{\frac{1}{2}}$. Note however that for a manifold with boundary and boundary defining function $x$

$$
\mathcal{C}^{\infty}\left(X ;{ }^{\mathrm{sc}} \Omega^{\frac{1}{2}}\right)=x^{-\frac{1}{2}(\operatorname{dim} X)} \mathcal{C}^{\infty}\left(X ; \Omega_{\mathrm{b}}^{\frac{1}{2}}\right)=x^{-\frac{1}{2}(\operatorname{dim} X+1)} \mathcal{C}^{\infty}\left(X ; \Omega^{\frac{1}{2}}\right)
$$

\section{BOUNDARY BICHARACTERISTICS}

If $g \in \rho_{\sigma}^{-2} \mathcal{C}^{\infty}\left({ }^{\mathrm{sc}} \bar{T}^{*} X\right)$ is the metric function and $f=\sqrt{g} \in \rho_{\sigma}^{-1} \mathcal{C}^{\infty}\left({ }^{\mathrm{sc}} \bar{T}^{*} X \backslash 0\right)$, then ${ }^{\mathrm{sc}} H_{f} \in \rho_{\partial} \mathcal{V}_{\mathrm{b}}\left({ }^{\mathrm{sc}} \bar{T}^{*} X \backslash 0\right)$ is the generator of geodesic flow. Thus, analyzing the integral curves of $f$, or $g$, amounts to examining the geodesic flow. Here we are interested in the behaviour of the geodesics near the boundary of $X$, rather than in the interior. Necessarily, in a product decomposition of the manifold near the boundary, with boundary defining function for which (0.1) holds,

$$
g=\tau^{2}+h(y, \mu)+x g^{\prime} \text { near }{ }^{\mathrm{sc}} T_{\partial X}^{*} X
$$

where $h(y, \mu)$ is the metric function on $T^{*} \partial X$ transferred to ${ }^{\mathrm{sc}} T_{\partial X}^{*} X$ using the defining function of the boundary, $x$, i.e. using the isomorphism

$$
\mu \cdot \frac{d y}{x} \longmapsto \mu \cdot d y \text {. }
$$

Lemma 2. For $0 \neq \lambda \in \mathbb{R}$ the integral curves of ${ }^{s c} H_{f}^{1,0}=\frac{1}{x} s c H_{f}$ on the characteristic variety

$$
\Sigma(\lambda)=\left\{\tau^{2}+h=\lambda^{2}\right\} \subset{ }^{s c} T_{\partial X}^{*} X
$$

are the points on the two connected components of the radial sub-variety, the incoming and outgoing parts, being respectively $G^{\sharp}(\lambda)$ and $G^{\sharp}(-\lambda)$ where (see Fig.2)

$$
G^{\sharp}(\lambda)=\{\tau=-\lambda, \mu=0, x=0\}
$$

and the curves of the form

$$
\begin{gathered}
\tau=|\lambda| \cos \left(s+s_{0}\right) \\
\mu=|\lambda| \sin \left(s+s_{0}\right) \hat{\mu} \\
(y, \hat{\mu})=\exp \left(\left(s+s_{0}\right) H_{\frac{1}{2} h}\right)\left(y^{\prime}, \hat{\mu}^{\prime}\right)
\end{gathered}
$$

where $s_{0} \in[0, \pi], s \in\left(-s_{0}, \pi-s_{0}\right),\left(y^{\prime}, \hat{\mu}^{\prime}\right) \in T^{*} \partial X, h\left(y^{\prime}, \hat{\mu}^{\prime}\right)=1$ and $d s / d t=$ $\frac{1}{2}|\mu|=\frac{1}{2} h(y, \mu)^{\frac{1}{2}}$.

Proof. The integral curves of $\frac{1}{x}{ }^{\mathrm{sc}} H_{f}$ are the same as those of $\frac{1}{x}{ }^{\mathrm{sc}} H_{g}$ near $\Sigma(\lambda)$, $\lambda \neq 0$. At $\partial X$ the rescaled Hamilton vector field is

$$
{ }^{\mathrm{sc}} H_{g}^{1,0}=2 \tau x \partial_{x}-2 h \partial_{\tau}+2 \tau \mu \cdot \partial_{\mu}+H_{h}(y, \mu) .
$$

Introducing polar coordinates with respect to the radial variable in $\mu$ :

$$
\hat{\mu}=h(y, \mu)^{-\frac{1}{2}} \mu,|\mu|=h(y, \mu)^{\frac{1}{2}}
$$

gives

$$
\frac{d}{d t} \hat{\mu}=-h_{y}^{\prime}(y, \hat{\mu})|\mu|, \frac{d}{d t} y=h_{\eta}^{\prime}(y, \hat{\mu})|\mu|
$$


Figure 2. The extended integral curves of ${ }^{\mathrm{sc}} H_{f}^{1,0}$ in $\tau$ and $\mu$ coordinates.

In terms of the new parameter $s$, satisfying $d s / d t=\frac{1}{2}|\mu|$ along the integral curves, this reduces to

$$
\begin{gathered}
\frac{d}{d s} \hat{\mu}=-\frac{1}{2} h_{y}^{\prime}(y, \hat{\mu}), \frac{d}{d s} y=\frac{1}{2} h_{\eta}^{\prime}(y, \hat{\mu}) \\
\frac{d \tau}{d s}=-|\mu| \text { and } \frac{d}{d s}|\mu|=\tau .
\end{gathered}
$$

Together, (3.9), (3.10) and the definition of $s$ give (3.5).

These uniform equations for the geodesics near the boundary permit a straightforward analysis of their global behaviour. For example the following result is the original motivation for the result conjectured in [10] which is the main theorem of this paper. Since it is not explicitly used below, we only sketch a proof.

Proposition 1. If a sequence of maximally extended geodesics in $X^{\circ}$, for a scattering metric, approaches the boundary uniformly then it has a subsequence converging to a geodesic in $\partial X$ of length $\pi$. Conversely any such geodesic segment is the limit of such a sequence of geodesics in the interior.

Proof. Suppose $\gamma_{i}(s)$ is a sequence of maximally extended geodesics in the interior of $X$ such that $\sup x\left(\gamma_{i}(s)\right) \rightarrow 0$ as $i \rightarrow \infty$. These are integral curves of the renormalized Hamilton vector field ${ }^{\mathrm{sc}} H_{g}^{2,0} \in \mathcal{V}_{\mathrm{b}}(Y), Y={ }^{\mathrm{sc}} \bar{T}^{*} X$. Since $Y$ is compact any subsequence must have a subsequence converging to a union of integral curves of ${ }^{\mathrm{sc}} H_{g}^{2,0}$ which, by assumption, must lie in the boundary. As noted in Lemma 2, these are points in $G^{\sharp}( \pm \lambda)$ or geodesics seqments of length $\pi$ over the boundary. From (3.6), $\tau$ is a monotone decreasing function if $h \neq 0$. The assumption that the curves in $X^{\circ}$ are maximally extended means that there must be limit points at which $\tau= \pm \lambda$, so the limit cannot consist of a single point in $G^{\sharp}( \pm \lambda)$. The converse is similarly straightforward.

\section{RESCALED LAGRANGIAN}

The 'Lagrangian' with which a generalized plane wave is associated turns out to be, in the general case, a pair of submanifolds of ${ }^{\mathrm{sc}} T_{\partial X}^{*} X$. For fixed $y \in \partial X$ and 
$0 \neq \lambda \in \mathbb{R}$ the main part of it is obtained as the union of the integral curves of ${ }^{\mathrm{sc}} H_{g}^{0,1}$ with limit point on $G^{\sharp}(\lambda)$ above $y$ :

$$
\begin{aligned}
G_{y}(\lambda)=\left\{\left(y^{\prime}, \tau, \mu\right) \in{ }^{\mathrm{sc}} T_{\partial X}^{*} X ;\right. & \tau^{2}+|\mu|^{2}=\lambda^{2}, \mu \neq 0, \\
& \left.\lim _{t \rightarrow \infty} \exp \left(\operatorname{sgn}(\lambda) t^{\mathrm{sc}} H_{g}^{0,1}\right)\left(y^{\prime}, \zeta\right)=(y,-\lambda, 0)\right\}
\end{aligned}
$$

where $\zeta=(\tau, \mu)$.

Proposition 2. For each $y \in \partial X$ and $0 \neq \lambda \in \mathbb{R}, G_{y}(\lambda) \subset{ }^{s c} T_{\partial X}^{*} X$ is a smooth submanifold with closure

$$
\overline{G_{y}(\lambda)}=G_{y}(\lambda) \sqcup\{(y,-\lambda, 0)\} \sqcup F_{\pi}(y, \lambda)
$$

which is smooth near $(y,-\lambda, 0)$ and has at most a conic singularity in $\mu$ at

$$
\begin{aligned}
F_{\pi}(y, \lambda)=\left\{\left(y^{\prime \prime}, \lambda, 0\right)\right. & { }^{s c} T_{\partial X}^{*} X ; \\
& \left.\exists \text { a geodesic of length } \pi \text { linking } y \text { and } y^{\prime \prime} \text { in } \partial X\right\} .
\end{aligned}
$$

Proof. From Lemma 2, $s \uparrow \pi$ as $t \rightarrow \infty$ (if we normalize $s_{0}$ to be 0 ). Hence, putting $r=\pi-s$ we see that $G_{y}(\lambda), \lambda>0$ (the case of $\lambda<0$ is analogous), is the union of curves $\mu^{\prime}=\lambda \sin r \hat{\mu}^{\prime}, \tau^{\prime}=-\lambda \cos r,\left(y^{\prime}, \mu^{\prime}\right)=\exp \left(-r H_{\frac{1}{2} h}\right)\left(y^{\prime}, \hat{\mu}\right),|\hat{\mu}|=1$. Since $\sin r$ is odd in $r$ it follows that $\overline{G_{y}(\lambda)}$ is smooth near $(\pi, y,-\lambda)$. Similarly it follows directly from (3.5) that $\overline{G_{y}(\lambda)}$ is smooth, near the other end, $s \downarrow 0$, of these curves when expressed in $\mu$ polar coordinates.

The boundary hypersurface, ${ }^{\mathrm{sc}} T_{\partial X}^{*} X$, of ${ }^{\mathrm{sc}} T^{*} X$ carries a natural contact structure. It arises in essentially the same way that the contact structure arises on the sphere bundle at infinity of the cotangent bundle in the boundaryless case. Thus, the canonical form is such that

$$
d^{\mathrm{sc}} \alpha=d\left(\frac{{ }^{\mathrm{sc}} \tilde{\chi}}{x}\right)
$$

where ${ }^{\mathrm{sc}} \tilde{\chi}$ is a smooth 1 -form on ${ }^{\mathrm{sc}} T^{*} X$ near the boundary. Indeed, in canonical coordinates $x, y, \tau, \mu$

$$
\begin{aligned}
& { }^{\mathrm{sc}} \alpha=\tau \frac{d x}{x^{2}}+\mu \cdot \frac{d y}{x} \\
& { }^{\mathrm{sc}} \tilde{\chi}=d \tau+\mu \cdot d y .
\end{aligned}
$$

Moreover, given the choice of defining function $x$, the pull-back ${ }^{\mathrm{sc}} \chi$ of ${ }^{\mathrm{sc}} \tilde{\chi}$ to ${ }^{\mathrm{sc}} T_{\partial X}^{*} X$ is uniquely determined. This fixes the contact structure on ${ }^{\mathrm{sc}} T_{\partial X}^{*} X$. If a general boundary defining function is used in place of $x$ then the resulting form is a positive multiple of ${ }^{\mathrm{sc}} \chi$, so the (oriented) contact line bundle is completely natural.

Lemma 3. The manifold $G_{y}(\lambda)$ is Legendre for the contact structure on ${ }^{s c} T_{\partial X}^{*} X$, that is, a submanifold of maximal dimension $(=\operatorname{dim} X-1)$ on which ${ }^{s c} \chi$ vanishes identically.

Proof. At the 'initial point' $(y,-\lambda, 0), d \tau=0$ on the tangent space to $G_{y}(\lambda)$ so it is clearly Legendre. In $T^{*} \partial X$ any submanifold, $\Lambda_{f}$, of the form

$$
(y, \hat{\mu})=\exp \left(s H_{\frac{1}{2} h}\right)\left(y^{\prime}, \hat{\mu}^{\prime}\right),|\mu|=f(s),
$$

where $f$ is a smooth function and $y^{\prime}$ is fixed but $s$ and $\hat{\mu}^{\prime}$ vary with $h\left(y^{\prime}, \hat{\mu}^{\prime}\right)=$ const, is Lagrangian. In fact, since $\Lambda_{1}$ is Lagrangian we only need to check that $\sum_{i} d y_{i} \wedge$ $\mu_{i} d s=0$ on $\Lambda_{1}$ that is that $\mu \cdot d y \wedge d s=0$. For a fixed $s=s_{0}, \Lambda_{1} \cap\left\{s=s_{0}\right\}$ is 
contained in $\exp \left(s_{0} H_{\frac{1}{2} h}\right)\left(T_{y^{\prime}}^{*} \partial X\right)$ which is a homogeneous Lagrangian submanifold. Hence $\mu \cdot d y$ vanishes there and consequently it is proportional to $d s$ on $\Lambda_{1}$.

Thus, from (3.5), the 2 -form $\sum_{i} \mu_{i} \wedge d y_{i}$ vanishes on $G_{y}(\lambda)$. Hence ${ }^{\mathrm{sc}} \chi$ is necessarily closed on $G_{y}(\lambda)$. Therefore there is, near $(y,-\lambda, 0)$, a unique smooth function $g$ with ${ }^{\text {sc }} \chi=d g$ on $G_{y}(\lambda)$ and $g(y,-\lambda, 0)=0$. The vector field ${ }^{\mathrm{sc}} H_{p}^{2,0}$ is Legendrian in the sense that

$$
{ }^{\mathrm{sc}} \chi\left(\left.{ }^{\mathrm{sc}} H_{p}^{2,0}\right|_{x=0}\right)=\left\langle d \tau+\mu \cdot d y,-2 h \partial_{\tau}+h_{\mu}^{\prime} \partial_{y}\right\rangle=-2 h+\mu \cdot h_{\mu}^{\prime} \equiv 0,
$$

where we have used (3.6) and the homogeneity of $h$ in $\mu$. Thus $g$ must be constant on $G_{y}(\lambda)$ which must in consequence be Legendrian near $(y,-\lambda, 0)$ and hence everywhere.

\section{INTERSECTING PAIRS OF LEGENDRE SUBMANIFOLDS WITH CONIC POINTS}

When the Legendre manifold $\overline{G_{y}(\lambda)}$ is singular at $F_{\pi}$, as is often the case, a second Legendre manifold passing through $F_{\pi}$ is needed to carry off the singularities of a generalized plane wave. This is the section, $G^{\sharp}(-\lambda)$ of ${ }^{\mathrm{sc}} T_{\partial X}^{*} X$ defined in (3.4) The union of these two surfaces is a singular Legendre variety

$$
\widetilde{G}_{y}(\lambda)=\overline{G_{y}(\lambda)} \cup G^{\sharp}(-\lambda) .
$$

Note that $G_{y}(\lambda)$ also meets $G^{\sharp}(\lambda)$, at the initial point $(0, y,-\lambda)$. At this intersection $\overline{G_{y}(\lambda)}$ is smooth so, in the construction of $P(\lambda)$, no singularities will propagate onto $G^{\sharp}(\lambda)$. Although, particularly in view of the push-forward result in Proposition 16 , it would be quite natural to include $G^{\sharp}(\lambda)$ as part of the Legendre variety we shall not do so.

In fact, we shall consider a somewhat wider class of Legendre manifolds with conic points obtained by abstraction of these conditions. Thus suppose that

$$
\widetilde{G}=\bar{G} \cup G^{\sharp} \subset{ }^{\mathrm{sc}} T_{\partial X}^{*} X
$$

is a closed set with $G^{\sharp} \subset{ }^{\mathrm{sc}} T_{\partial X}^{*} X$ a finite union of global sections determined by one defining function:

$$
G^{\sharp}=\bigcup_{j} G^{\sharp}\left(\bar{\lambda}_{j}\right), G^{\sharp}\left(\bar{\lambda}_{j}\right)=\operatorname{graph}\left\{-\bar{\lambda}_{j} \frac{d x}{x^{2}}\right\} \subset{ }^{\mathrm{sc}} T_{\partial X}^{*} X .
$$

In the decomposition (5.2) we suppose that $G$ is a smooth Legendrian submanifold with closure $\bar{G}$ and that

$$
\bar{G} \backslash G \subset \operatorname{sp}\left\{\frac{d x}{x^{2}}\right\},(\bar{G} \backslash G) \cap \operatorname{sp}\left\{\frac{d x}{x^{2}}\right\} \subset \bar{G} \cap G^{\sharp}
$$

is the site of an at most conic singularity of $\bar{G}$. That is, we assume that $\bar{G}$ is the image in ${ }^{\mathrm{sc}} T_{\partial X}^{*} X$ of a smooth manifold, $\widehat{G}$, in $\mu$-polar coordinates. In the local coordinates $y, \tau, \mu$ this means

$$
\bar{G}=\left\{(\tau, y, \mu) ; \tau=T(y, \hat{\mu},|\mu|), g_{j}(y, \hat{\mu},|\mu|)=0\right\}, \hat{\mu}=\mu /|\mu|
$$

where the $n$ functions $g_{1}, \ldots, g_{n}$ are such that $d_{(y, \hat{\mu})} g_{j}, j=1, \ldots, n$ are independent at the base point $(\bar{y}, \hat{\bar{\mu}})$. In particular this means that $|\mu|$ has non-vanishing differential on $\widehat{G}$, where locally

$$
\widehat{G}=\left\{(y, \tau, r, \hat{\mu}) ; r \geq 0,|\hat{\mu}|=1, \tau=T(y, \hat{\mu}, r), g_{j}(y, \hat{\mu}, r)=0\right\} .
$$


Notice that we do not exclude the possibility, as indeed occurs in the some examples here (for instance Euclidean space), that $\bar{G}$ may be smooth at some points of intersection with $G^{\sharp}$.

Definition 1. By an intersecting pair of Legendre submanifolds with conic points we shall mean a pair $\widetilde{G}=\left(G, G^{\sharp}\right)$ satisfying the conditions (5.2) - (5.6).

The description of conic singularities can be given a direct global interpretation in terms of blow up along the submanifold

$$
\operatorname{sp}\left\{\frac{d x}{x^{2}}\right\}=\{x=\mu=0\}
$$

in ${ }^{\mathrm{sc}} T^{*} X$. As remarked above, over the boundary $\operatorname{sp}\left\{d x / x^{2}\right\}$ is determined by the choice of defining function $x$ up to constant multiple and quadratic terms, and hence by $G^{\sharp}$. It is a 'scattering conormal bundle' to $\partial X$, since it consists of the multiples of $d x / x^{2}=-d\left(\frac{1}{x}\right)$. The description in terms of blow up also reveals the relationship between ${ }^{\mathrm{sc}} T_{\partial X}^{*} X$ and $T^{*} \partial X$.

First recall (see for instance [9]) that if $G \subset Y$ is a closed embedded submanifold of a $\mathcal{C}^{\infty}$ manifold $Y$ then the blow up of $Y$ along $G,[Y ; G]$, is defined as follows. As a set it is the disjoint union

$$
[Y ; G]=(Y \backslash G) \sqcup S N G .
$$

Here, $S N G$ is the spherical normal bundle to $G$ in $Y$; thus if $N G=T_{G} Y / T G$ is the normal bundle to $G$ then $S N G=(N G \backslash 0) / \mathbb{R}_{+}$with $\mathbb{R}_{+}$acting on the fibres. The blow-down map, $\beta:[Y ; G] \rightarrow Y$ is defined to be the identity on $Y \backslash G$ and the projection to the base on $S N G$. The $\mathcal{C}^{\infty}$ structure, $\mathcal{C}^{\infty}([Y ; G])$, on $[Y ; G]$ is obtained by adjoining to the lift of $\mathcal{C}^{\infty}(Y)$ the lifts of $\rho$ and functions of the form $f / \rho$; here

$$
\rho=\left(\sum_{i} g_{i}^{2}\right)^{\frac{1}{2}}
$$

where the $g_{i} \in \mathcal{C}^{\infty}(Y)$ collectively define $G$ and $f \in \mathcal{C}^{\infty}(Y)$ vanishes on $G$. Since $\mathcal{C}^{\infty}([Y ; G])$ includes the lifts of all $\mathcal{C}^{\infty}$ functions on $Y$, the blow down map $\beta$ is smooth. When $Y$ is a manifold with boundary and $G$ is a closed embedded submanifold of the boundary then $[Y ; G]=(Y \backslash G) \cup S N^{+} G$ where $S N^{+} G$ is the (closed) inward-pointing part of the spherical normal bundle. In either case the set $\beta^{-1}(Y)$ by which $Y$ is replaced in the blow up is a boundary hypersurface, which is sometimes called the front face and denoted $\mathrm{ff}[Y ; G]$; the lift of $\rho$ is a defining function for this new boundary hypersurface. The reader is reminded that this is simply a uniform, and invariant, way of introducing polar coordinates in $Y$ around $G$. Using this notation we introduce the blown up manifold

$$
\beta:{ }^{\mathrm{sc}} \widehat{T}^{*} X \equiv\left[{ }^{\mathrm{sc}} T^{*} X, \operatorname{sp}\left\{\frac{d x}{x^{2}}\right\}\right] \longrightarrow{ }^{\mathrm{sc}} T^{*} X .
$$

Under this blow up the boundary hypersurface ${ }^{\mathrm{sc}} T_{\partial X}^{*} X$ lifts to one of the boundary hypersurfaces which is just the blown up manifold:

$$
\beta_{\partial}:{ }^{\mathrm{sc}} \widehat{T}_{\partial X}^{*} X \equiv\left[{ }^{\mathrm{sc}} T_{\partial X}^{*} X, \operatorname{sp}\left\{\frac{d x}{x^{2}}\right\}\right] \longrightarrow{ }^{\mathrm{sc}} T_{\partial X}^{*} X .
$$

When no confusion is likely to arise the same notation will be used for these two blow down maps, $\beta$. 
The conditions (5.5) and (5.6) can now be rephrased in terms of the blown up manifold and the lift of $\bar{G}$ to ${ }^{\mathrm{sc}} \widehat{T}_{\partial X}^{*} X$. Namely

$$
\begin{gathered}
\widehat{G}=\beta_{\partial}^{*} \bar{G}=\operatorname{cl} \beta_{\partial}^{-1}\left(\bar{G} \backslash G^{\sharp}\right) \text { in }{ }^{\mathrm{sc}} \widehat{T}_{\partial X}^{*} X \text { is an embedded submanifold } \\
\text { with boundary, intersecting } \partial\left({ }^{\mathrm{sc}} \widehat{T}_{\partial X}^{*} X\right) \text { transversally. }
\end{gathered}
$$

There is a further observation concerning this blow up which will be useful later. Given a choice of boundary defining function $x$, the front face of ${ }^{\mathrm{sc}} \widehat{T}^{*} X$ fibres over the variable $\tau$, which is the coefficient of $d x / x^{2}$, and each of the fibres is naturally isomorphic to the compactification, $\bar{T}^{*} \partial X$, of the cotangent bundle to the boundary. Since the components of $G^{\sharp}$ are by assumption $\tau$-fibres the inverse images $\beta^{-1} G^{\sharp}\left(\lambda_{i}\right)$ (which we denote as the lifts $\beta^{*} G^{\sharp}\left(\lambda_{i}\right)$ ) of these components are each naturally isomorphic, with isomorphism $\gamma$, to $\bar{T}^{*} \partial X$. If $S^{*} \partial X=\partial \bar{T}^{*} \partial X$ is the sphere bundle (at infinity) it follows that

$$
\gamma: \beta_{\partial}^{*} G^{\sharp}\left(\lambda_{i}\right)=\partial\left(\beta^{*} G^{\sharp}\left(\lambda_{i}\right)\right) \longrightarrow S^{*} \partial X .
$$

In fact, the isomorphism (5.11) can be seen from the analogous discussion for the restricted blow-up ${ }^{\mathrm{sc}} \widehat{T}_{\partial X}^{*} X$ where the boundary of ${ }^{\mathrm{sc}} \widehat{T}_{\partial X}^{*} X$ fibres over $\tau$ which each fiber isomorphic to $S^{*} \partial X$. See the proof of Lemma 12 for the local coordinate form of the maps.

Proposition 3. If $\widetilde{G}$ is an intersecting pair of Legendre submanifolds with conic points then for each component $G^{\sharp}\left(\lambda_{i}\right)$ of $G^{\sharp}$

$$
\gamma\left(\beta^{*} G \cap \beta^{*} G^{\sharp}\left(\lambda_{i}\right)\right) \subset S^{*} \partial X
$$

is a Legendrian submanifold with respect to the standard contact structure. Consequently there is a unique homogeneous Lagrangian submanifold, $\Lambda\left(\widetilde{G}, \lambda_{i}\right) \subset T^{*} \partial X$ such that

$$
\Lambda\left(\widetilde{G}, \lambda_{i}\right) \cap S^{*} \partial X=\gamma\left(\beta^{*} G \cap \beta^{*} G^{\sharp}\left(\lambda_{i}\right)\right) .
$$

Proof. Consider the boundary $\widehat{G}_{0}=\partial \widehat{G}$ of the blown up form of $\bar{G}$. By the assumption (5.4) each component of it lies in a fixed $\tau$ fibres of the front face of ${ }^{\mathrm{sc}} \widehat{T}_{\partial X}^{*} X$ and has dimension $\operatorname{dim} X-2=\operatorname{dim} \partial X-1$. Since $G$ is Legendre the scattering contact form ${ }^{\mathrm{sc}} \tilde{\chi}=d \tau+\mu \cdot d y$ in (4.5) vanishes on it. On blowing up, $\widehat{\eta}=\mu /|\mu|, \tau$, $y$ and $\rho=|\mu|$ give local coordinates so

$$
d \tau=\rho \widehat{\eta} \cdot d y
$$

must vanish on $\widehat{G}_{0}$, which is defined by $\rho=0$ in $\widehat{G}$. Differentiating (5.13) it follows that $d \rho \wedge \widehat{\eta} \cdot d y=0$ at $\widehat{G}_{0}$ on $\widehat{G}$ and hence that $\widehat{\eta} \cdot d y=0$ on $\widehat{G}_{0}$ which is therefore a Legendre submanifold of $S^{*} \partial X$.

Notice that $\Lambda(\widetilde{G}, \lambda)$ is a Lagrangian associated to any value of $\lambda$ at which $\widetilde{G} \cap$ $G^{\sharp}(\lambda) \neq \emptyset$ provided $\widetilde{G}$ satisfies the conditions of a conic Lengendre variety nearby. This includes the case of smooth points. These conic Lagrangian manifolds can be thought of as providing 'boundary conditions' for the Legendre manifold $\widetilde{G}$.

The Legendre manifold $G_{y}(\lambda)$ corresponds to a single plane wave, emanating from the point $y$ on the boundary. The carrier of the singularities of the kernel of the Poisson operator is the Legendre manifold which consists of the whole family of the $G_{y}(\lambda)$. Thus we consider the compact manifold with boundary $X \times \partial X$. The lift 
of the function $x \in \mathcal{C}^{\infty}(X)$ is still a defining function for the boundary so, abusing notation slightly, we write

$$
G^{\sharp}(\lambda)=\operatorname{graph}\left\{-\lambda \frac{d x}{x^{2}}\right\} \subset{ }^{\mathrm{sc}} T_{\partial X \times \partial X}^{*}(X \times \partial X)
$$

which is Legendre. For the definition of the scattering symbol map, $j$, used in the next proposition see [10] or $\S 10$ below.

Proposition 4. For each $0 \neq \lambda \in \mathbb{R}$ there exists a unique intersecting pair of Legendre submanifolds with conic points $\widetilde{G}(\lambda)=\left(G(\lambda), G^{\sharp}(-\lambda)\right)$ in ${ }^{s c} T_{\partial(X \times \partial X)}^{*}(X \times$ $\partial X)$ such that

$$
\begin{gathered}
\left.j\left(\Delta-\lambda^{2}\right)\right|_{G(\lambda)}=0 \text { and } \\
\Lambda(\widetilde{G}(\lambda), \lambda)=N^{*} \Delta \subset T^{*}(\partial X \times \partial X) .
\end{gathered}
$$

This pair satisfies

$$
\Lambda(\widetilde{G}(\lambda), \lambda)=\left\{\left(m, m^{\prime}\right) \in T^{*} \partial X \times T^{*} \partial X ; m=\exp \left(\pi H_{\frac{1}{2} h}\left(m^{\prime}\right)\right\}^{\prime}\right.
$$

where $\Delta \subset \partial X \times \partial X$ is the diagonal, and $\left(y, \eta ; y^{\prime}, \eta^{\prime}\right)^{\prime}=\left(y, \eta ; y^{\prime},-\eta^{\prime}\right)$.

Proof. The discussion of $\S 3$ can be applied in this product case. It should be noted however that even though the new symbol $g^{\prime}=j\left(\Delta_{X}\right)$ is still given by (3.1) in product coordinates $x, y, y^{\prime}, \tau, \mu, \mu^{\prime}$, the rescaled Hamilton vector field is not as in (3.6) because of the implicit $x$ dependence in $\mu^{\prime}$. Rather it is

$$
2 \tau x \partial_{x}-2 h \partial_{\tau}+2 \tau \mu \cdot \partial_{\mu}+2 \tau \mu^{\prime} \cdot \partial_{\mu^{\prime}}+H_{h}(y, \mu)
$$

Thus $\mu^{\prime}$ simply scales under the flow. Consider the submanifold

$$
\begin{aligned}
& \left\{\left(y, y^{\prime} ; \tau, \mu, \mu^{\prime}\right) ;(y, \hat{\mu})=\exp \left(s H_{\frac{1}{2} h}\right)\left(y^{\prime}, \hat{\mu}^{\prime}\right), \tau=\lambda \cos s,\right. \\
& \left.\mu=\lambda \sin s \hat{\mu}, \mu^{\prime}=-\lambda \sin s \hat{\mu}^{\prime}\right\} \subset{ }^{\mathrm{sc}} T_{\partial(X \times \partial X)}^{*}(X \times \partial X) .
\end{aligned}
$$

The same argument as in the proof of Lemma 3 shows that this submanifold is Legendrian and satisfies the two conditions in (5.15). Clearly it also satisfies (5.16).

Conversely (5.15) implies that

$$
\pi_{L}\left(G(\lambda) \cap \pi^{-1}(X \times\{y\})\right)=G_{y}(\lambda), \forall y \in \partial X,
$$

where $\pi_{L}:{ }^{\mathrm{sc}} T_{\partial(X \times \partial X)}^{*}(X \times \partial X) \rightarrow{ }^{\mathrm{sc}} T_{\partial X}^{*} X$ is the left projection. Hence $(5.15)$ determines $\widetilde{G}(\lambda)$ uniquely.

Notice that $\widetilde{G}(\lambda)$ is essentially the union of the $\widetilde{G}_{y}(\lambda)$ except for the behaviour of the variables $\mu^{\prime}$.

\section{Parametrization AND Equivalence}

The local parametrization of smooth Legendre submanifolds of ${ }^{\mathrm{sc}} T_{\partial X}^{*} X$ is completely analogous to, in fact is equivalent to, the parametrization of conic Lagrangian submanifolds (i.e. Legendre submanifolds of the cosphere bundle) in the boundaryless case. The only formal difference is the relation of the 'radial vector field' to the base; this results in parametrizations requiring between 0 and $\operatorname{dim} X-1$ parameters rather than between 1 and $\operatorname{dim} X$ parameters as in the conic case.

To make the analogy as clear as possible we shall extend the given Legendre submanifold, $G \subset{ }^{\mathrm{sc}} T_{\partial X}^{*} X$, to a Lagrangian submanifold of $T^{*} X$, near $\partial X$, which 
is $x$-translation-invariant as a submanifold of ${ }^{\mathrm{sc}} T^{*} X$. This can be accomplished by the choice of a product decomposition near the boundary and then taking

$$
\Lambda_{G}=\left\{(x, y, \xi, \eta) \in T^{*} X ; 0<x<\epsilon,\left(y, x^{2} \xi, x \eta\right) \in G\right\} .
$$

Note that $\Lambda_{G}$ is Lagrangian if and only if $G$ is Legendre, since the tangency of $\partial_{x}$ (as a vector field in the coordinates $x, y, \tau, \mu$ ) to $\Lambda_{G}$ implies that ${ }^{\mathrm{sc}} \tilde{\chi}=0$ on $\Lambda_{G}$.

By a parametrization of $G$ (or $\Lambda_{G}$ ) near some point $(\bar{\tau}, \bar{y}, \bar{\mu}) \in G$, we mean a function $\varphi(y, u)$, defined and $\mathcal{C}^{\infty}$ near $(\bar{y}, \bar{u}) \in \partial X \times \mathbb{R}^{k}$, satisfying the normalization and non-degneracy conditions

$$
\begin{gathered}
\varphi(\bar{y}, \bar{u})=-\bar{\tau}, d_{y} \varphi(\bar{y}, \bar{u})=\bar{\mu}, d_{u} \varphi(\bar{y}, \bar{u})=0 \\
d_{(y, u)} \frac{\partial \varphi}{\partial u_{i}}, i=1, \ldots k \text { are independent at }(\bar{y}, \bar{u})
\end{gathered}
$$

and parametrizing the submanifold in the sense that

$$
\begin{aligned}
C_{\varphi}=\left\{(y, u) ; d_{u} \varphi(y, u)\right. & =0\} \ni(y, u) \longmapsto \\
& \left\{\left(y,-\varphi(y, u), d_{y} \varphi(y, u)\right)=(y, \tau, \mu)\right\}
\end{aligned}
$$

is a diffeomorphism from a neighbourhood of $(\bar{y}, \bar{u})$

$$
\text { to a neighbourhood of }(\bar{y}, \bar{\tau}, \bar{\mu}) \text { in } G \text {. }
$$

Notice that the true 'phase function' parametrizing $\Lambda_{G}$ is

$$
\Phi(x, y, u)=\frac{\varphi(y, u)}{x}
$$

which is homogeneous of degree -1 in $x$. The parametrization condition (6.1.iii) then becomes more transparent:

$$
d \Phi=-\varphi \frac{d x}{x^{2}}+\frac{d \varphi}{x}=\tau \frac{d x}{x^{2}}+\mu \cdot \frac{d y}{x} \text { if } d_{u} \varphi=0 .
$$

Two parametrizations $\varphi_{1}(y, u)$ and $\varphi_{2}\left(y, u^{\prime}\right)$ are equivalent near the base points $(\bar{y}, \bar{u})$ and $\left(\bar{y}, \bar{u}^{\prime}\right)$ if there is a family of local diffeomorphism of $\mathbb{R}^{k}$, depending smoothly on $y$ as a parameter, for $y$ near $\bar{y}$,

$$
U_{y}: \mathbb{R}^{k} \longrightarrow \mathbb{R}^{k}, \quad U_{\bar{y}}(\bar{u})=\bar{u}^{\prime}
$$

such that $\varphi_{2}\left(y, U_{y}(u)\right)=\varphi_{1}(y, u)$ near $\bar{y}, \bar{u}$.

The local equivalence of parametrizations is very much as in the standard case, see especially Theorems 3.1.3 and 3.1.6 of [5].

Proposition 5. Any Legendre submanifold, $G$, of $T_{\partial X}^{*} X$, has a local parametrization near each point with any number

$$
k \geq k_{\min }=\operatorname{dim}\left(T_{(\bar{y}, \bar{\tau}, \bar{\mu})} G \cap\{d y=0\}\right)
$$

of parameters and with Hessian $\partial_{u}^{2} \varphi(\bar{y}, \bar{u})$ having exactly $p$ positive eigenvalues for any $0 \leq p \leq k-k_{\min }$. Two such parametrizations based at $(\bar{y}, \bar{\tau}, \bar{\mu})$ are equivalent if and only if they have the same number of parameters and $\partial_{u}^{2} \varphi$ has the same signature at the base point.

Proof. For a given base point $(\bar{y}, \bar{\tau}, \bar{\mu}) \in G$ we construct a minimal parametrization, one with $k=k_{\min }$ in (6.6). By a linear change of $y$ variables it can be arranged that

$$
T_{(\bar{y}, \bar{\tau}, \bar{\mu})} G \cap\{d y=0\}=\left\{\left(0 ; 0, \mu_{1}, \ldots, \mu_{k}, 0, \ldots, 0\right)\right\}
$$


where, by definition, $k=k_{\min }$. Since $d \tau+\mu \cdot d y=0$ on $G$,

$$
\sum_{i} d \mu_{i} \wedge d y_{i}=0
$$

there and thus the differentials $d y_{1}, \ldots, d y_{k}$ vanish on $T_{(\bar{\tau}, \bar{y}, \bar{\mu})} G$. It follows that $\mu^{\prime}=\left(\mu_{1}, \ldots, \mu_{k}\right)$ and $y^{\prime \prime}=\left(y_{k+1}, \ldots, y_{n-1}\right)$ together give local coordinates on $G$ near $(\bar{\tau}, \bar{y}, \bar{\mu})$, which is therefore of the form

$$
G=\left\{\tau=T\left(y^{\prime \prime}, \mu^{\prime}\right), y^{\prime}=Y^{\prime}\left(y^{\prime \prime}, \mu^{\prime}\right), \mu^{\prime \prime}=\mu^{\prime \prime}\left(y^{\prime \prime}, \mu^{\prime}\right)\right\} .
$$

Consider

$$
\varphi\left(y, \mu^{\prime}\right)=y^{\prime} \cdot \mu^{\prime}-T\left(y^{\prime \prime}, \mu^{\prime}\right)-Y^{\prime}\left(y^{\prime \prime}, \mu^{\prime}\right) \cdot \mu^{\prime} .
$$

This parametrizes $G$, since $d T=-\mu \cdot d Y^{\prime}-\mu^{\prime \prime}$. Thus $\partial_{\mu^{\prime}} \varphi=0 \Longleftrightarrow y^{\prime}=Y^{\prime}\left(y^{\prime \prime}, \mu^{\prime}\right)$ and then $\mu^{\prime}=d_{y^{\prime}} \varphi, \mu^{\prime \prime}=d_{y^{\prime \prime}} \varphi$. Relabelling the $\mu^{\prime}$ variables as $u$ and reverting to the original coordinates gives a minimal parametrization of $(\bar{\tau}, \bar{y}, \bar{\mu})$. A general parametrization, as in the statement of the proposition, can be obtained by adding to $\varphi$ a non-degenerate quadractic form in additional $u$ variables.

Equivalence of parametrizations can be shown essentially as in [5], Theorem 3.1.6. Since the only difference is the absence of homogeneity in the parameters, the details are omitted.

\section{CONIC PAIRS AND THEIR PARAMETRIZATION}

We also need to produce similar parametrizations for Legendre submanifolds with conic singularities.

By a parametrization of $\widetilde{G}$ near a singular point $(\bar{y}, \bar{\tau}, 0, \hat{\bar{\mu}}) \in \widehat{G}_{0}=\partial \widehat{G}$ we mean a $\mathcal{C}^{\infty}$ function $\varphi(y, s, u)$ defined near $(\bar{y}, 0,0)$ in $\partial X \times[0, \infty) \times \mathbb{R}^{k-1}$ of the form

$$
\varphi(y, s, u)=-\bar{\tau}+s \psi(y, s, u), \bar{\tau}=\text { const }
$$

such that

$$
d_{(y, u)} \psi \text { and } d_{(y, u)} \frac{\partial \psi}{\partial u_{j}}, j=1, \ldots, k \text { are independent at }(\bar{y}, 0,0)
$$

and for which the map

$$
C_{\varphi}=\left\{(y, s, u) ; \frac{\partial \varphi}{\partial s}=0, \frac{\partial \psi}{\partial u}=0, s \geq 0\right\} \longmapsto\left(y,-\varphi,\left|d_{y} \varphi\right|, \frac{d_{y} \varphi}{\left|d_{y} \varphi\right|}\right) .
$$

is a diffeomorphism onto a neighbourhood of $(\bar{y}, \bar{\tau}, 0, \hat{\bar{\mu}})$ into $\widehat{G}$.

Proposition 6. Every intersecting pair of Legendre submanifolds with conic points, ${ }^{\text {in }}{ }^{s c} T_{\partial X}^{*} X$, in the sense of $(5.2)-(5.6)$ admits a parametrization at each point of $\widehat{G}_{0}$.

Proof. By making a linear change of coordinates in $y$, and by scaling $x$, it can be assumed that $\hat{\bar{\mu}}=(0, \ldots, 0,1)$ at the base point. Thus the projective variables $\hat{\mu}_{j}=\mu_{j} / \mu_{n-1}, j=1, \ldots, n-2$, are smooth on $\widehat{G}$ near the base point. By making a further linear change of variables it can be ensured that $d \hat{\mu}_{1}, \ldots, d \hat{\mu}_{k-1}, d \mu_{n-1}$ are independent, on $\widehat{G}$, at the base point, with $d \hat{\mu}_{k}, \ldots, d \hat{\mu}_{n-2}$ vanishing there. As shown in the proof of Lemma $3, d \hat{\mu} \wedge d y^{\prime}=0$ on $\widehat{G}_{0}$ which implies that $d y_{1}, \ldots, d y_{k-1}=0$ at the base point. Hence

$$
\hat{\mu}^{\prime}=\left(\hat{\mu}_{1}, \ldots, \hat{\mu}_{k-1}\right), y^{\prime \prime}=\left(y_{k}, \ldots, y_{k-2}\right) \text { and } \mu_{n-1}
$$


give local coordinates on $\widehat{G}$ near the base point.

A parametrizing function is now given by

$$
\varphi(y, s, u)=s y^{\prime} \cdot u-T\left(y^{\prime \prime}, s, y\right)-s Y^{\prime}\left(y^{\prime \prime}, s, u\right) \cdot u+y_{n-1} s-Y_{n-1}\left(y^{\prime \prime}, s, u\right) s
$$

where $\widehat{G}=\left\{\tau=T\left(y^{\prime \prime}, \mu_{n-1}, \hat{\mu}^{\prime}\right), y^{\prime}=Y^{\prime}\left(y^{\prime \prime}, \mu_{n-1}, \hat{\mu}^{\prime}\right), \hat{\mu}^{\prime \prime}=M^{\prime \prime}\left(y^{\prime \prime}, \mu_{n-1}, \hat{\mu}^{\prime}\right)\right\}$.

Since $\tau=\bar{\tau}$ on $\widehat{G}_{0}=\left\{\mu_{n-1}=0\right\}, T\left(y^{\prime \prime}, s, u\right)=\bar{\tau}+s T^{\prime}\left(y^{\prime \prime}, s, u\right)$, so $\varphi$ in $(7.5)$ is of the form (7.1). Furthermore,

$$
\begin{aligned}
d \varphi= & \left(y^{\prime} \cdot u-\frac{\partial T}{\partial s}-\frac{\partial}{\partial s}\left(s Y^{\prime} \cdot u\right)+y_{n-1}-\partial_{s}\left(Y_{n-1} s\right)\right) d s \\
& +s\left(y^{\prime}-\partial_{u} T-\partial_{u}\left(Y^{\prime} u\right)-\partial_{u}\left(Y_{n-1}\right)\right) \cdot d u \\
& +s u \cdot d y^{\prime}+s d y_{n-1}-M^{\prime \prime} \cdot d y^{\prime \prime}
\end{aligned}
$$

where $M^{\prime \prime}=\partial_{y^{\prime \prime}} T+s u \cdot \partial_{y^{\prime \prime}} Y^{\prime}+s \partial_{y^{\prime \prime}} Y_{n-1}$. The independence condition (7.2) is then clear and (7.3) follows readily from the fact that $\widehat{G}$ is Legendre.

The parametrization given in Proposition 6 also gives a parametrization of the Lagrangian submanifolds $\Lambda(\widetilde{G}, \bar{\lambda})$ given by Proposition 3 . In fact, in a conic neighbourhood of $(\bar{y}, \overline{\hat{\mu}}), \Lambda(\widetilde{G}, \bar{\lambda})$ is parametrized by

$$
\begin{gathered}
\phi(y, \theta)=\theta_{1} \psi\left(y, 0, \theta^{\prime} / \theta_{1}\right),\left(\theta_{1}, \theta^{\prime}\right) \in \Gamma \subset \mathbb{R}^{k+1}, \\
\Gamma \text { a conic neighbourhood of }(1,0, \cdots, 0) \in \mathbb{R}^{k+1} .
\end{gathered}
$$

We also need to consider the equivalence of such parametrizations. This follows essentially as in the standard case. Two parametrizations $\varphi_{1}(y, s, u)$ and $\varphi_{2}\left(y, s^{\prime}, u^{\prime}\right)$ are said to be equivalent near the base point $(\bar{y}, 0,0)$ if there is a family of local diffeomorphims, $U_{y}$, of $\mathbb{R}_{+} \times \mathbb{R}^{k-1}$, mapping $\{0\} \times \mathbb{R}^{k-1}$ to itself, depending smoothly on $y \in \partial X$ as a parameter and such that, $U_{\bar{y}}(0,0)=(0,0)$ and near $(\bar{y}, 0,0)$,

$$
\varphi_{2}\left(y, U_{y}(s, u)\right)=\varphi_{1}(y, s, u) .
$$

Proposition 7. Two parametrizations of a conic Legendre pair, $\varphi_{1}$ and $\varphi_{2}$, based at $(\bar{y}, \bar{\tau}, \bar{\mu})$, are equivalent if and only if they have the same number of parameters and

$$
\operatorname{sgn} \partial_{u}^{2} \psi_{1}(y, 0,0)=\operatorname{sgn} \partial_{u}^{2} \psi_{2}(y, 0,0),
$$

where $\varphi_{i}(y, s, u)=-\bar{\tau}+s \psi_{i}(y, s, u)$, for $i=1,2$.

Proof. As already noted this result can be deduced by following the proof of Theorem 3.1.6 of [5]. We shall therefore only indicate the points at which the argument is slightly different. Consider the two parametrizations $\phi_{1}$ and $\phi_{2}$ of $\Lambda(G, \bar{\lambda})$ obtained from $\varphi_{1}$ and $\varphi_{2}$ as in (7.7). Since they parametrize the same Lagrangian and

$$
\partial_{\theta}^{2} \phi_{i}(\bar{y} ; 1,0)=\left(\begin{array}{cc}
0 & 0 \\
0 & \partial_{u}^{2} \psi_{i}(\bar{y}, 0,0)
\end{array}\right)
$$

Theorem 3.1.6 of [5] shows them to be equivalent. Thus we can assume that $\psi_{1}(y, 0, u)=\psi_{2}(y, 0, u)$.

Proceeding as in the first step of the proof in [5] we can also assume that $\varphi_{1}$ and $\varphi_{2}$ are equal to second order on the set $C_{\varphi_{1}}$, where $C_{\varphi_{1}}$ is defined by (7.3). 
The independence of $d_{(y, u)} \psi_{1}$ and $d_{(y, u)}\left(\psi_{1 u_{j}}^{\prime}\right)$, for $j=1, \cdots, k-1$, and Taylor's formula give

$$
\begin{aligned}
\varphi_{2}(y, s, u)=\varphi_{1} & (y, s, u)+\tilde{a}_{00}(y, u, s)\left(\psi_{1}+s \psi_{1 s}^{\prime}\right)^{2} \\
& \sum_{i=1}^{k-1} \tilde{a}_{0 i}(y, s, u)\left(\psi_{1}+s \psi_{1 s}^{\prime}\right) \psi_{1 u_{i}}^{\prime}+\sum_{i, j=1}^{k-1} \tilde{a}_{i j}(y, s, u) \psi_{1 u_{i}}^{\prime} \psi_{1 u_{j}}^{\prime} .
\end{aligned}
$$

Since $\psi_{1}=\psi_{2}$ at $s=0$ and $\varphi_{i}=-\bar{\tau}+s \psi_{i}$, it can be arranged that $\tilde{a}_{i j}=s a_{i j}$ with the $a_{i j}$ smooth. Hence the analogue of $B$ in (3.18) of [5] is small for $s$ small and thus the second step of the proof there can be applied. This yields the desired parameter change $U_{y}$.

\section{Oscillating Functions}

The simplest type of Legendre submanifold of ${ }^{\mathrm{sc}} T_{\partial X}^{*} X$ is a section, $G$, given by the graph of the differential of $\varphi / x, \varphi \in \mathcal{C}^{\infty}(\partial X)$. Then $\exp (i \varphi / x) \in \mathcal{C}^{-\infty}(X)$ is a typical Legendre distribution associated to $G$. In particularly with the notion of wave front set defined in [10] (this is briefly recalled in $\S 10$ below)

$$
{ }^{\mathrm{sc}} \mathrm{WF}\left(e^{i \varphi / x}\right)=G \text {. }
$$

In this case we define

$$
I_{\mathrm{sc}}^{m}\left(X, G ;{ }^{\mathrm{sc}} \Omega^{\frac{1}{2}}\right)=e^{i \varphi / x} x^{m+\frac{n}{4}} \cdot \mathcal{C}^{\infty}\left(X ;{ }^{\mathrm{sc}} \Omega^{\frac{1}{2}}\right), \quad n=\operatorname{dim} X .
$$

The normalization here is arranged, as is discussed below, to conform to the convention of Hörmander in [5] for the order of a Lagrangian distribution.

One of the most fundamental examples of such an oscillating function is the kernel of the Fourier transform. Since we need to consider coordinate-invariance below, consider the invariant Fourier transform from half-densities on a vector space $V$ to half-density on the dual $V^{*}$

$$
\mathcal{F}\left(u|d v|^{\frac{1}{2}}\right)=\left(\int e^{-i\left\langle v, v^{*}\right\rangle} u(v)|d v|\right)\left|d v^{*}\right|^{\frac{1}{2}} .
$$

The Schwartz kernel

$$
e^{-i\left\langle v, v^{*}\right\rangle}|d v|^{\frac{1}{2}}\left|d v^{*}\right|^{\frac{1}{2}} \in \mathcal{C}^{-\infty}\left(\bar{V} \times \bar{V}^{*} ;{ }^{\mathrm{sc}} \Omega^{\frac{1}{2}}\right)
$$

is completely well-defined, where $\bar{V}$ is the stereographic compactification of $V$. We are most interested in points in the interior of $V^{*}$ and near the boundary of $\bar{V}$ so we shall just consider $\bar{V} \times V^{*}$, a manifold with boundary. Applying the definition, (8.2), we find

Lemma 4. For any real vector space $V$

$$
e^{-i\left\langle v, v^{*}\right\rangle}|d v|^{\frac{1}{2}}\left|d v^{*}\right|^{\frac{1}{2}} \in I_{s c}^{0}\left(\bar{V} \times V^{*}, \mathcal{L} ;{ }^{s c} \Omega^{\frac{1}{2}}\right)
$$

where $\mathcal{L}=\operatorname{graph}\left\{-d\left\langle v, v^{*}\right\rangle\right\} \subset{ }^{s c} \bar{T}^{*}{ }_{\partial \bar{V} \times V^{*}}\left(\bar{V} \times V^{*}\right)$ is the Legendre relation.

Proof. Notice that the order is 0 since $|d v|^{\frac{1}{2}}\left|d v^{*}\right|^{\frac{1}{2}} \in x^{\frac{1}{2} \operatorname{dim} V} \mathcal{C}^{\infty}\left(\bar{V} \times V^{*} ;{ }^{s c} \Omega^{\frac{1}{2}}\right)$.

To justify calling this the Legendre relation consider linear coordinates $\eta$ in $V$ and dual coordinates $y$ in $V^{*}$. If $\eta=\theta / x, x=1 /|\eta|$, then $\partial \bar{V} \simeq \mathbb{S}^{n-1}$ with coordinates $\theta$. Let $x, \theta, y, \tau, \mu^{\prime}, \mu^{\prime \prime}$ be the corresponding coordinates in ${ }^{\mathrm{sc}} T^{*}\left(\bar{V} \times V^{*}\right)$. Thus

$$
-d\left\langle v, v^{*}\right\rangle=-d \frac{\theta \cdot y}{x}=\theta \cdot y \frac{d x}{x^{2}}-(y-(y \cdot \theta) \theta) \cdot \frac{d \theta}{x}-\frac{\theta}{x} d y
$$


so $\mathcal{L}$ is the submanifold

$$
\tau=y \cdot \theta, \mu^{\prime}=-y+(y \cdot \theta) \theta, \mu^{\prime \prime}=-\theta .
$$

The two projections $\left(\theta, y, \tau, \mu^{\prime}, \mu^{\prime \prime}\right) \longmapsto\left(\theta, \tau, \mu^{\prime}\right)$ and $\left(\theta, y, \tau, \mu^{\prime}, \mu^{\prime \prime}\right) \longrightarrow\left(y, \mu^{\prime \prime}\right)$ are the coordinate forms of invariantly defined projections

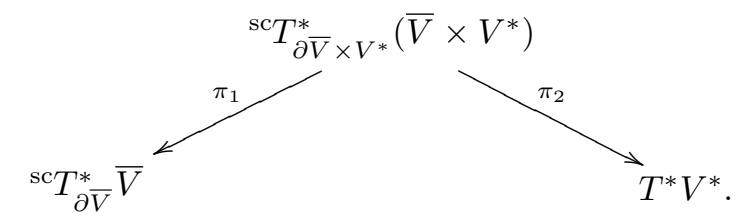

We also introduce $S^{*} V^{*}=\partial \bar{T}^{*} V^{*}$, the cosphere bundle of $V^{*}$, thought of as the sphere at infinity. Directly from (8.7) we find

Lemma 5. Under the two projections (8.8), $\mathcal{L}$ defines the 'Legendre diffeomorphism'

$$
\begin{gathered}
L:{ }^{s c} T_{\partial \bar{V}}^{*} \bar{V} \longrightarrow S^{*} V^{*}, \\
L\left(\theta ; \tau, \mu^{\prime}\right)=\left(y, \mu^{\prime \prime}\right) \Longleftrightarrow\left(\theta, y ;-\tau,-\mu^{\prime}, \mu^{\prime \prime}\right) \in \mathcal{L}, L\left(\theta, \tau, \mu^{\prime}\right)=\left(\mu^{\prime}-\tau \theta,-\theta\right) .
\end{gathered}
$$

The Legendre transformation, $L$ in (8.9), is a contact diffeomorphism of ${ }^{\mathrm{sc}} T_{\partial \bar{V}}^{*} \bar{V}$ onto $S^{*} V^{*}$. It is therefore natural to expect the Fourier transform to act as a (local) Fourier integral operator transforming the Legendre distributions associated to $G \subset{ }^{\mathrm{sc}} T_{\partial X}^{*} X$ to Lagrangian distributions in the ordinary sense. Indeed we shall ruthlessly exploit this transformation.

\section{Maslov bundle}

The Maslov bundle is defined over any conic Lagrangian submanifold $\Lambda \subset$ $T^{*} Y \backslash 0$, for any manifold $Y$. For $l \in \Lambda$, a conic Lagrangian, let $G_{l}$ be the part of the Grassmanian of Lagrange planes in $T_{l}\left(T^{*} Y\right)$ consisting of the planes transversal to both $T_{l} \Lambda$ and the fibre $T_{l}\left(T_{\pi(l)}^{*} Y\right) \cong T_{\pi(l)}^{*} Y$. The fibre, $M_{l}$, of the Maslov bundle consists of the locally constant functions on $G_{l}$, with values in $\mathbb{C}$, satisfying the transition condition between components

$$
f\left(p_{l}\right)=i^{m\left(p_{l}, p_{l}^{\prime} ; T_{l} G, T_{\pi(l)}^{*} Y\right)} f\left(p_{l}^{\prime}\right)
$$

where $m\left(p_{l}, p_{l}^{\prime} ; T_{l} \Lambda, T_{\pi(l)}^{*} Y\right) \in \mathbb{Z}$ is the Maslov index, defined for any four Lagrange planes with the first two transversal to the second two.

This bundle is completely natural under coordinate transformations, i.e. if $F^{*}$ : $T^{*} Y_{2} \longrightarrow T^{*} Y_{1}$ is the pull-back map of a (local) diffeomorphism $F: Y_{1} \longrightarrow Y_{2}$ then for any conic Lagrangian $\Lambda_{1} \subset T^{*} Y_{2} \backslash 0$ the Maslov bundle $M\left(\Lambda_{2}\right)$ over $\Lambda_{2}=$ $\left(F^{*}\right)^{-1}\left(\Lambda_{1}\right)$ is canonically identified with $\left(F^{*}\right)^{*} M\left(\Lambda_{1}\right)$, the pull-back of the Maslov bundle over $\Lambda_{1}$.

This naturality allows us to define the Maslov bundle on a Legendre submanifold $\Gamma \subset{ }^{\text {sc }} T_{\partial X}^{*} X$ by local identification with the conic Lagrangian case. Thus, near any point on $\partial X, X$ can be locally identified with $\bar{V}$, the compactification of a vector space of the same dimension. The map (8.9) identifies $\Gamma$, locally, with a Legendre submanifold of $S^{*} V^{*}$. The Maslov bundle over the associated conic Lagrangian submanifold of $T^{*} V^{*} \backslash 0$ is induced by its restriction to the Legendre manifold at infinity. This identification therefore fixes the fibres of the Maslov bundle over $\Gamma$ 
near the chosen base point. However, two local coordinate identifications of $X$ with $\bar{V}_{1}$, and $\bar{V}_{2}$ induce Legendre transformation $L_{1}$ and $L_{2}$ such that $L_{1} L_{2}^{-1}$ is the lift of a coordinate transformation from $V_{2}^{*}$ to $V_{1}^{*}$. The naturality of the Maslov bundle under coordinate transformations means that the Maslov bundle $M$ over $\Gamma$ is well-defined, with the properties

$$
M_{l}(\Gamma)=L^{*} M_{L(l)}(L(\Gamma)) \quad \text { locally. }
$$

It is also possible to define the Maslov bundle intrinsically on $\Gamma$, but we do not have to do so.

In the case of a pair of Legendre submanifolds with conic points, notice that the tangent planes to $\widehat{G}$, even at points of $\widehat{G}_{0}$, are Legendre planes in the tangent space to ${ }^{\mathrm{sc}} T_{\partial X}^{*} X$. Thus the construction about extends to define the Maslov bundle over $\widehat{G}$, and hence over the whole of $\widetilde{G}$.

\section{Pseudodifferential operators And Symbols}

The algebra of scattering pseudodifferential operators on a general compact manifold with boundary is defined, in [10], by reference to the model space, $\mathbb{S}_{+}^{n}$, thought of as the stereographic compactification of $\mathbb{R}^{n}$. To give maximal invariance properties to the symbol we consider operators on sections of the bundle ${ }^{\mathrm{sc}} \Omega^{\frac{1}{2}}$. Under stereographic projection, $\mathrm{SP}: \mathbb{R}^{n} \hookrightarrow \mathbb{S}_{+}^{n}$, the Lebesgue half-density $|d z|^{\frac{1}{2}}$ becomes a non-vanishing smooth section of ${ }^{\mathrm{sc}} \Omega^{\frac{1}{2}}$.

Thus, in the model case, $A \in \Psi_{\mathrm{sc}}^{m, k}\left(\mathbb{S}_{+}^{n} ;{ }^{\mathrm{sc}} \Omega^{\frac{1}{2}}\right)$ if and only if $A^{\prime}=\mathrm{SP}^{*} \cdot A \cdot\left(\mathrm{SP}^{-1}\right)^{*}$ is of the form

$$
\begin{gathered}
A^{\prime}\left(u(z)|d z|^{\frac{1}{2}}\right)=(2 \pi)^{-n} \int e^{i\left(z-z^{\prime}\right) \cdot \zeta} a\left(\frac{z+z^{\prime}}{2}, \zeta\right) u\left(z^{\prime}\right) d z^{\prime} d \zeta|d z|^{\frac{1}{2}} \\
\text { with } a(z, \zeta)=\mathrm{SP}_{2}^{*} a^{\prime}, a^{\prime} \in \rho_{\sigma}^{-m} \rho_{\partial}^{k} \mathcal{C}^{\infty}\left(\mathbb{S}_{+}^{n}\right) .
\end{gathered}
$$

Here $\mathrm{SP}_{2}=\mathrm{SP} \times \mathrm{SP}: \mathbb{R}_{z}^{n} \times \mathbb{R}_{\zeta}^{n} \longrightarrow \mathbb{S}_{+}^{n} \times \mathbb{S}_{+}^{n}, \rho_{\sigma} \in \mathcal{C}^{\infty}\left(\mathbb{S}_{+}^{n} \times \mathbb{S}_{+}^{n}\right)$ is a defining function for $\mathbb{S}_{+}^{n} \times \mathbb{S}^{n-1}$ and $\rho_{\partial} \in \mathcal{C} \infty\left(\mathbb{S}_{+}^{n} \times \mathbb{S}_{+}^{n}\right)$ is a defining function for $\mathbb{S}^{n-1} \times \mathbb{S}_{+}^{n}$. In fact the definition in [10] was in terms of left quantization, not Weyl quantization as in (10.1), but these are completely equivalent. (See [6] or $\S 18.5$ of [7]).

The manifold $\mathbb{S}_{+}^{n} \times \mathbb{S}_{+}^{n}$, together with its identification with $\mathbb{R}_{z}^{n} \times \mathbb{R}_{\zeta}^{n}=T^{*} \mathbb{R}^{n}$ in the interior, is canonically isomorphic to ${ }^{\mathrm{sc}} \bar{T}^{*} \mathbb{S}_{+}^{n}$. The joint symbol of $A$, as defined in [10], is the equivalence class of

$$
\begin{gathered}
a^{\prime} \in \rho_{\sigma}^{-m} \rho_{\partial}^{k} \mathcal{C}^{\infty}\left({ }^{\mathrm{sc}} \bar{T}^{*} \mathbb{S}_{+}^{n}\right) \\
\bmod \rho_{\sigma}^{-m} \rho_{\partial}^{k+1} \mathcal{C}^{\infty}\left({ }^{\mathrm{sc}} \bar{T}^{*} \mathbb{S}_{+}^{n}\right)+\rho_{\sigma}^{-m+1} \rho_{\partial}^{k} \mathcal{C}^{\infty}\left({ }^{\mathrm{sc}} \bar{T}^{*} \mathbb{S}_{+}^{n}\right),
\end{gathered}
$$

and as such is independent of coordinates.

Since the operators in $\Psi_{\mathrm{sc}}^{m, k}\left(\mathbb{S}_{+}^{n},{ }^{\mathrm{sc}} \Omega^{\frac{1}{2}}\right)$ act on half-densities, much more is true:

Lemma 6. For any compact manifold with boundary the joint symbol of $A \in$ $\Psi_{s c}^{m, k}\left(X ;{ }^{s c} \Omega^{\frac{1}{2}}\right), j(A)$, is well-defined as an element of

$$
\rho_{\sigma}^{-m} \rho_{\partial}^{k} \mathcal{C}^{\infty}\left({ }^{s c} \bar{T}^{*} X\right) \quad \bmod \rho_{\sigma}^{-m+2} \rho_{\partial}^{k+2} \mathcal{C}^{\infty}\left({ }^{s c} \bar{T}^{*} X\right)
$$

by $a^{\prime}$ in (10.1) in any local identification of ${ }^{s c} \bar{T}^{*} X$ with ${ }^{s c} \bar{T}^{*} \mathbb{S}_{+}^{n}$ resulting from a local identification of $X$ and $\mathbb{S}_{+}^{n}$.

Proof. This follows from the definition and the coordinate transformation properties of the Weyl symbol (see [6] or $§ \S 18.2$ and 18.5 of [7]). 
The $\mathbb{R}^{+}$action on the fibres of ${ }^{\mathrm{sc}} T^{*} X$ is independent of coordinates. This allows the joint symbol to be identified near the boundary, $\partial\left({ }^{\mathrm{sc}} \bar{T}^{*} X^{\circ}\right)$, of the compactified fibres over the interior as the sum of homogeneous functions

$$
j(A)=\sigma_{m}(A)+\sigma_{m-1}^{\mathrm{sub}}(A) \text { near } \partial\left({ }^{\mathrm{sc}} \bar{T}^{*} X^{\circ}\right) .
$$

Thus the subprincipal symbol becomes a function on $T^{*} X^{\circ} \backslash 0$ which is homogeneous of degree $m-1$. The part of the symbol we are most interested in is that near ${ }^{s c} T_{\partial X}^{*} X$, where there is no natural transversal $\mathbb{R}^{+}$action. Then there is no natural splitting analogous to (10.4). However the choice of a boundary defining function, $x$, for $X$ and of a product decomposition of $X$ near $\partial X$ does give a splitting. Near, but not at, the boundary

$$
{ }^{\mathrm{sc}} T^{*} X={ }^{\mathrm{sc}} T^{*}(0, \epsilon) \times x^{-1} T^{*} \partial X \text { with }{ }^{\mathrm{sc}} T^{*}(0, \epsilon)=(0, \epsilon)_{x} \times \mathbb{R}_{\tau}
$$

where the basis element $d x / x^{2}$ of ${ }^{{ }^{s c}} T^{*}(0, \epsilon)$ is used to define the coordinate $\tau$. This means that, for $A \in \Psi_{\mathrm{sc}}^{m, k}\left(X ;{ }^{\mathrm{sc}} \Omega^{\frac{1}{2}}\right)$,

$$
j(A)=x^{k} p_{k}+x^{k+1} p_{k+1}, p_{k}, p_{k+1} \in \mathcal{C}^{\infty}\left(\mathbb{R}_{\tau} \times T^{*} \partial X\right) .
$$

This decomposition will be used in computations below. In particular the rescaled Hamiltion vector field of $j(A)$ can be written

$$
{ }^{\mathrm{sc}} H_{j(A)}^{*, k}=\partial_{\tau} p_{k}\left(x \partial_{x}+R_{\mu}\right)-\left(R_{\mu} p_{k}+k p_{k}\right) \partial_{\tau}+H_{p_{k}}+x W, W \in \mathcal{V}_{\mathrm{b}} \text { near }{ }^{\mathrm{sc}} T_{\partial X}^{*} X
$$

where $H_{p_{k}}$ is the Hamilton vector field of $p_{k}$ as a function on $T^{*} \partial X, R_{\mu}$ is the radial vector field in the tangential directions, $\rho_{\partial}=x$ and the rescaling at $\rho_{\sigma}=0$ has been dropped.

It is very convenient below to reduce microlocal questions near ${ }^{\mathrm{sc}} T_{\partial X}^{*} X$ to 'conventional' microlocal analysis near $\bar{T}^{*} \mathbb{R}^{n}$. This is accomplished by the Fourier transform.

Proposition 8. For any vector space $V$ the intrinsic Fourier transform (8.5) defines, by conjugation, an isomorphism

$$
C_{F}: \Psi_{s c}^{m, k}\left(\bar{V} ;{ }^{s c} \Omega^{\frac{1}{2}}\right) \longrightarrow \Psi_{s c}^{-k,-m}\left(\bar{V}^{*} ;{ }^{s c} \Omega^{\frac{1}{2}}\right)
$$

such that if $\tilde{L}:{ }^{s c} \bar{T}^{*} \bar{V} \cong \bar{V} \times \bar{V}^{*} \longrightarrow \bar{V}^{*} \times \bar{V} \cong s c \bar{T}^{*} \bar{V}^{*}$ is the map $\tilde{L}(\bar{v}, \bar{w})=$ $\left(\bar{w}, \mathrm{SP}\left(-\mathrm{SP}^{-1}(\bar{v})\right)\right)$ then

$$
j\left(C_{F} A\right)=\tilde{L}^{*} j(A) .
$$

Proof. The intrinsic Fourier transform, (8.3), is defined by conjugation with stereographic projection $V \hookrightarrow \bar{V}$. Thus (10.8) reduces to the covariance of the Weyl calculus under Fourier transformation. Namely if $A^{\prime}$ is given by (10.1), with $A=\mathrm{SP}^{*} \cdot A \cdot\left(\mathrm{SP}^{-1}\right)^{*}$ then $C_{F}(A)=\mathrm{SP}^{*} \cdot A^{\prime \prime} \cdot\left(\mathrm{SP}^{-1}\right)^{*}$ where

$$
\begin{aligned}
A^{\prime \prime} v(\tau)= & (2 \pi)^{-n} \int e^{i \tau z+i\left(z-z^{\prime}\right) \zeta-i z^{\prime} \cdot \tau^{\prime}} a\left(\frac{z+z^{\prime}}{\tau}, \zeta\right) v\left(\tau^{\prime}\right) d \tau^{\prime} d z^{\prime} d z d \zeta \\
& =(2 \pi)^{-n} \int e^{i\left(\tau-\tau^{\prime}\right) \cdot Z} a\left(Z,-\frac{\tau+\tau^{\prime}}{2}\right) v\left(\tau^{\prime}\right) d \tau^{\prime} d z
\end{aligned}
$$

This gives (10.9). 
We remark that the Legendre diffeomorphism $L$ defined in Lemma 5 is the boundary value of $\tilde{L}$ :

$$
L=\left.\tilde{L}\right|_{\mathrm{sc}_{\bar{T}}{ }_{\partial \bar{V}} \bar{V}}:{ }^{\mathrm{sc}} \bar{T}_{\partial \bar{V}}^{*} \bar{V} \longrightarrow S^{*} \bar{V} .
$$

It is instructive to obtain the somewhat non-obvious (from this point of view) coordinate expression (8.9) from the definition of $\tilde{L}$.

The Laplacian on a Riemann manifold acts naturally on the sections of the half-density bundle since the Riemannian density gives a trivalizing section.

Lemma 7. For the Laplacian $\Delta \in \operatorname{Diff}_{s c}^{2}\left(X ;{ }^{s c} \Omega^{\frac{1}{2}}\right) \subset \Psi_{s c}^{2,0}\left(X ;{ }^{s c} \Omega^{\frac{1}{2}}\right)$ of a scattering metric the joint symbol, including subprincipal terms, is the equivalence class of the metric function $g \in \rho_{\sigma}^{-2} \mathcal{C}^{\infty}\left(s c \bar{T}^{*} X\right)$.

Proof. This follows directly from Lemma 3 in [10].

We also note the form, for scattering pseudodifferential operators, of the 'oscillatory testing' definition of the symbol.

Lemma 8. If $\varphi \in \mathcal{C}^{\infty}(X)$ has $d\left(\left.\varphi\right|_{\partial X}\right) \neq 0$ or $\left.\varphi\right|_{\partial X} \neq 0$ on the support of $\nu \in$ $\mathcal{C}^{\infty}\left(X ;{ }^{s c} \Omega^{\frac{1}{2}}\right)$ and $x \in \mathcal{C}^{\infty}(X)$ is a boundary defining function then

$$
\begin{gathered}
A\left(e^{i \varphi / x} \nu\right)=e^{i \varphi / x} x^{k} \nu^{\prime}, \nu^{\prime} \in \mathcal{C}^{\infty}\left(X ;{ }^{s c} \Omega^{\frac{1}{2}}\right) \\
x^{k} \nu^{\prime}-\left.j(A)\right|_{\operatorname{graph}\left(d \frac{\varphi}{x}\right)} \nu \in x^{k+1} \mathcal{C}^{\infty}\left(X ;{ }^{s c} \Omega^{\frac{1}{2}}\right)
\end{gathered}
$$

for any $A \in \Psi_{s c}^{m, k}\left(X ; \Omega^{\frac{1}{2}}\right)$. Moreover if $A$ is microlocally of order $k=+\infty$ near a point of $\operatorname{graph}(d(\varphi / x))$ over the boundary then $\nu^{\prime}$ and all its derivatives vanish rapidly with $x$ near the projection of that point in $\partial X$.

This can be proved directly from the definitions. A proof based on the conjugation properties of the Fourier transform is given in $\S 11$.

For completeness we note, without proof, the relationship between the scattering wave front set, ${ }^{\mathrm{sc}} \mathrm{WF}$, and the better known frequency set of semi-classical analysis, FS, (see [4]). Recall from [10] the definition of ${ }^{\mathrm{sc}} \mathrm{WF}(u)$ for any element $u \in \mathcal{C}^{-\infty}(X)$

$$
{ }^{\mathrm{sc}} \mathrm{WF}(u)=\bigcap\left\{\{j(A)=0\} ; A \in \Psi_{\mathrm{sc}}^{0,0}(X), A u \in \dot{\mathcal{C}}^{\infty}(X)\right\} \subset \partial^{\mathrm{sc}} \bar{T}^{*} X .
$$

Proposition 9. Let $X$ be a compact manifold with boundary and suppose that $u \in \mathcal{C}^{-\infty}(X)$ is smooth in the interior. If $F:[0, \epsilon) \times \partial X \longrightarrow X$ is a product decomposition of $X$ near the boundary then interpreting $F^{*} u=u(h, y)$ as a function of $y \in \partial X$ depending on the 'semi-classical parameter' $h \in(0, \epsilon)$

$$
\mathrm{FS}\left(B^{*} u\right)=\pi^{s c} W F(u),
$$

where $\pi:{ }^{s c} T_{\partial X}^{*} X \rightarrow T^{*} \partial X, \pi(y ; \tau, \mu)=(y, \mu)$, is the map determined by the choice of the defining function $x=h$ and product decomposition.

In this sense the scattering wavefront set is a refinement of the frequency set which unifies it with the standard wavefront set in the interior.

\section{LEGENDRE DISTRIBUTIONS}

Let $G \subset{ }^{\mathrm{sc}} T_{\partial X}^{*} X$ be a smooth (not necessarily closed) scattering Legendre submanifold. Near each point $p \in G$ we have shown that there exist local parametrizations of $G, \varphi \in \mathcal{C}^{\infty}\left(U \times U^{\prime}\right)$ where $\pi(p) \in U \subset \partial X$ and $U^{\prime} \subset \mathbb{R}^{k}$ is open in the 
parameter space. The Legendre distributions of order $m$, defined with respect to this local parametrization, are the functions with compound asymptotics

$$
\begin{gathered}
v(x, y)=(2 \pi)^{-\frac{n}{4}-\frac{k}{2}} \int e^{i \varphi(y, u) / x} a(x, y, u) x^{m-\frac{k}{2}+\frac{n}{4}} d u, \\
a \in \mathcal{C}_{c}^{\infty}\left([0, \epsilon) \times U \times U^{\prime}\right) .
\end{gathered}
$$

Definition 2. For a smooth Legendre submanifold

$$
G \subset{ }^{s c} T_{\partial X}^{*} X, u \in I_{s c}^{m}\left(X, G ;{ }^{s c} \Omega^{\frac{1}{2}}\right) \subset \mathcal{C}^{-\infty}\left(X ;{ }^{s c} \Omega^{\frac{1}{2}}\right)
$$

if, for any $\psi \in \mathcal{C}_{c}^{\infty}(X)$,

$$
\psi u=u_{0}+\sum_{j=1}^{J} v_{j} \cdot \nu_{j}
$$

where $u_{0} \in \dot{\mathcal{C}}^{\infty}\left(X ;{ }^{s c} \Omega^{\frac{1}{2}}\right), \nu_{j} \in \mathcal{C}^{\infty}\left(X ;{ }^{s c} \Omega^{\frac{1}{2}}\right)$ and the $v_{j}$ are, for $j=1, \ldots, J$, functions of the form (11.1) for parametrizations of $G$.

It is easy to see the coordinate invariance of these spaces of Legendre distributions. Namely if $f: X \longrightarrow X$ is a diffeomorphism and

$$
{ }^{\mathrm{sc}} f^{*}:{ }^{\mathrm{sc}} T^{*} X \longrightarrow{ }^{\mathrm{sc}} T^{*} X
$$

is the induced isomorphism then

$$
f^{*}: I_{\mathrm{sc}}^{m}\left(X, G ;{ }^{\mathrm{sc}} \Omega^{\frac{1}{2}}\right) \longrightarrow I_{\mathrm{sc}}^{m}\left(X,{ }^{\mathrm{sc}} f^{*} G ;{ }^{\mathrm{sc}} \Omega^{\frac{1}{2}}\right)
$$

is an isomorphism. To see this it suffices to show that if $v$ is of the form (11.1) for a parametrization of $G$ then $f^{*} v$ is of the same form for a parametrization of ${ }^{\mathrm{sc}} f^{*} G$. Let $f\left(x^{\prime}, y^{\prime}\right)=(x, y)$ be the induced change of local coordinates, so $x=x^{\prime} f_{0}\left(x^{\prime}, y^{\prime}\right)$, $y=Y\left(x^{\prime}, y^{\prime}\right)$ with $f_{0}>0$. Then

$$
f^{*} v=\int e^{i \varphi^{\prime}\left(y^{\prime}, u\right) / x^{\prime}} a^{\prime}\left(x^{\prime}, y^{\prime}, u\right)\left(x^{\prime}\right)^{m-\frac{k}{2}+\frac{n}{4}} d u
$$

where

$$
\varphi^{\prime}\left(y^{\prime}, u\right)=\varphi\left(Y\left(0, y^{\prime}\right), u\right) / f_{0}\left(0, y^{\prime}\right)
$$

and

$$
a^{\prime}\left(x^{\prime}, y^{\prime}, u\right)=a\left(x^{\prime} f_{0}\left(x^{\prime}, y^{\prime}\right), Y\left(x^{\prime}, y^{\prime}\right), u\right) \exp \left(i \frac{\varphi\left(Y\left(x^{\prime}, y^{\prime}\right), u\right)}{x^{\prime} f_{0}\left(x^{\prime}, y^{\prime}\right)}-i \frac{\varphi^{\prime}\left(y^{\prime}, u\right)}{x^{\prime}}\right)
$$

is smooth up to $x^{\prime}=0$. Since $\varphi^{\prime}\left(y^{\prime}, u\right) / x^{\prime}$ is clearly a parametrization of ${ }^{\mathrm{sc}} f^{*} G$ the coordinate invariance, (11.5), follows.

In fact, if $\varphi_{j}$ for $j=1, \ldots, N$, give local parametrizations of $G$ covering an open subset of $X$ then any element of $I^{m}\left(X, \Omega\right.$; $\left.^{\mathrm{sc}} \Omega^{\frac{1}{2}}\right)$ with support in that subset has a decomposition (11.3) for those parametrizations. It is quite straightforward to prove this and to develop the symbol calculus of these Legendre distributions by analogy with the usual case of the Lagrangian distributions of Hörmander. Rather than do so we shall use the (local) Fourier transform to reduce the discussion to this case. As already noted this reduction is based on the following result for the Fourier transform. 
Proposition 10. If $\bar{V}$ is the stereographic compactificiation of a vector space and $G \subset{ }^{s c} T_{\partial X}^{*} \bar{V}$ is a Legendre submanifold then (compactified) Fourier transformation gives an isomorphism

$$
{ }^{s c} \mathcal{F}: I_{s c}^{m}\left(\bar{V}, G ;{ }^{s c} \Omega^{\frac{1}{2}}\right) \longrightarrow I^{-m}\left(V, \Lambda ; \Omega^{\frac{1}{2}}\right) \cap \mathcal{E}^{\prime}\left(V ; \Omega^{\frac{1}{2}}\right)+\mathcal{S}\left(V ; \Omega^{\frac{1}{2}}\right),
$$

where $\Lambda$ is the unique homogeneous Lagrangian such that $\Lambda \cap S^{*} V=L(G)$.

Proof. By the coordinate invariance, (11.5), of these spaces it suffices to take $\bar{V}=$ $\mathbb{S}_{+}^{n}$. Let $\theta=z /|z|, x=1 /|z|$ be inverted polar coordinates. In these coordinates $v$, in (11.1), has Fourier transform

$$
{ }^{\mathrm{sc}} \mathcal{F} v(\zeta)=(2 \pi)^{-\frac{n}{4}-\frac{k}{2}} \int e^{-i \frac{\theta}{x} \cdot \zeta+i \varphi(\theta, u) / x} a(x, \theta, u) x^{m-\frac{k}{2}+\frac{n}{4}} d u \cdot \frac{d x d \theta}{x^{n+1}}|d \zeta|^{\frac{1}{2}}
$$

Written in terms of the linear coordinates $z=\theta / x$ this is of the form

$$
{ }^{\mathrm{sc}} \mathcal{F} v(\zeta)=(2 \pi)^{-\frac{n}{4}-\frac{k}{2}} \int e^{i \psi(\zeta, z, U)} b(\zeta, z, U) d z d U|d \zeta|^{\frac{1}{2}}
$$

where $U=|z| u$ and

$$
b(\zeta, z, U)=a\left(\frac{1}{|z|}, \frac{z}{|z|}, \frac{U}{|z|}\right)|z|^{-m-\frac{k}{2}-\frac{n}{4}} .
$$

Clearly $b$ is a 1-step polyhomogeneous symbol. Moreover,

$$
\psi(\zeta, z, U)=-z \cdot \zeta+\varphi\left(\frac{z}{|z|}, \frac{U}{|z|}\right)|z|
$$

parametrizes $\Lambda$. Thus, directly from the definition of Lagrangian distributions, ${ }^{\text {sc }} \mathcal{F} v \in I^{-m}\left(V, \Lambda ; \Omega^{\frac{1}{2}}\right)$. Necessarily its singular support is compact and the mapping property (11.9) easily follows.

This argument can be reversed, showing that (11.9) is an isomorphism.

We now briefly outline the proof of Lemma 8 from the previous section. Since the statement is local we can assume that $X=\bar{V}$. By Proposition $10,{ }^{\mathrm{sc}} \mathcal{F}\left(e^{i \phi / x} \nu\right)$ is a Lagrangian distribution associated to $\Lambda_{\phi} \subset T^{*} V^{*} \backslash 0$. In terms of projective variables it is parametrized by $z_{1}^{-1} \phi\left(z^{\prime} / z_{1}\right)-\langle\zeta, z\rangle$ where $z_{1}=1 / x, z^{\prime}=y / x$ and $\zeta=\left(\zeta_{1}, \zeta^{\prime}\right)$ are the corresponding dual variables. The statement $(10.11)$ then follows from the standard formula for the application of a pseudodifferential operator to a Lagrangian distribution (see Theorem 25.2.4 in [7], though in this case it is particularly simple) and from the proof of Proposition 10.

Before using this local result to discuss the symbolic properties of Legendre disitributions we shall consider the invariance properties of the local Fourier transform.

Proposition 11. Under a local diffeomorphism $G: U, p \longrightarrow U^{\prime}, p$ where $U, U^{\prime} \subset \mathbb{S}_{+}^{n}$ are open and $p, p^{\prime} \in \mathbb{S}^{n-1}=\partial \mathbb{S}_{+}^{n}$, the operator

$$
G_{\mathcal{F}}={ }^{s c} \mathcal{F} \circ G^{*} \circ \chi \circ{ }^{s c} \mathcal{F}^{-1}
$$

where $\chi \in \mathcal{C}_{c}^{\infty}\left(U^{\prime}\right)$, is a Fourier integral operator of order 0 on $\mathbb{R}^{n}$ associated with the (local) canonical diffeomorphism $L_{G}=L G^{*} L^{-1}$ of $T^{*} \mathbb{R}^{n}$ and having symbol $L^{*} \chi$ under the natural trivializations of Maslov and density bundles. 
Proof. As an operator on half-densities $G_{\mathcal{F}}$ has kernel

$$
(2 \pi)^{n} \int e^{-i \zeta \cdot z+G(z) \cdot \zeta^{\prime}}\left|\frac{\partial G}{\partial z}\right|^{\frac{1}{2}} \chi(z) d z|d \zeta|^{\frac{1}{2}}
$$

This has the symbolic properties as stated.

\section{Symbol CAlCulus}

For a Legendre distribution, associated to a smooth Legendre submanifold $G \subset$ ${ }^{\mathrm{sc}} T_{\partial X}^{*} X$, the transformation (11.9) reduces the definition of the symbol to the standard conic Lagrangian case.

If $Y$ is a manifold without boundary and $\Lambda \subset T^{*} Y \backslash 0$ is a conic Lagrangian the symbol mapping of Hörmander

$$
\sigma_{m}: I^{m}\left(Y, \Lambda ; \Omega^{\frac{1}{2}}\right) \longrightarrow S^{m+\frac{n}{4}}\left(\Lambda ; \Omega^{\frac{1}{2}} \otimes M\right)
$$

takes values in the homogeneous sections, of degree $m+\frac{n}{4}$, of $\Omega^{\frac{1}{2}} \otimes M$. If $\Gamma \subset S^{*} Y$ is the Legendre manifold associated to $\Lambda$, with $S^{*} Y=\partial \bar{T}^{*} Y$ the boundary of the fibrewise stereographic compactification, then (12.1) can be replaced by

$$
\begin{gathered}
\sigma_{m}^{\sharp}: I^{m}\left(Y, \Lambda ; \Omega^{\frac{1}{2}}\right) \longrightarrow \mathcal{C}^{\infty}\left(\Gamma ; E^{-m}(\Gamma)\right) \\
\text { where } E^{-m}(\Gamma)=\left|N^{*} \partial \bar{T}^{*} Y\right|^{-m-\frac{n}{4}} \otimes M_{\Gamma} \otimes \Omega_{\Gamma}^{\frac{1}{2}} .
\end{gathered}
$$

Notice that $M$, as a bundle over $\Lambda$, is canonically trivial over the $\mathbb{R}^{+}$lines and so induces a bundle $M_{\Gamma}$ over $\Gamma$. The power of the conormal bundle of infinity in (12.2) models a section which is homogeneous of degree $m+\frac{n}{4}$ and a smooth half-density on $\Gamma$ defines a unique smooth half-density on $\Lambda$ which is homogeneous of degree 0 .

Following Hörmander in [5] the symbol in (12.1) is defined as follows. Any element $u \in I^{m}\left(Y, \Lambda ; \Omega^{\frac{1}{2}}\right)$ is (by definition) a locally finite superposition of distributions defined by oscillatory integrals:

$$
u=(2 \pi)^{-\frac{n}{4}-\frac{N}{2}} \int e^{i \varphi(\zeta, \theta)} a(\zeta, \theta) d \theta|d \zeta|^{\frac{1}{2}}
$$

where $\zeta_{1}, \ldots, \zeta_{n}$ are local coordinates in $Y, a$ is a polyhomogeneous (that is, classical) symbol of order $m+\frac{n}{4}-\frac{N}{2}$, there are $N$ phase variables $\theta_{1}, \ldots, \theta_{N}$ and $\varphi$ is a local parametrization of $\Lambda$ over the support of $a$. The parametrization, $\varphi$, defines a local isomorphism (near the support of $a$ )

$$
p_{\varphi}: C_{\varphi}=\left\{(\zeta, \theta) ; \varphi_{\theta}^{\prime}=0\right\} \longrightarrow\left\{\left(\zeta, d_{\zeta} \phi\right) \in \Lambda\right\}
$$

and the symbol of $u$ in the sense of (12.1) is then locally on $\Lambda$

$$
\sigma_{m}(u)=\left(p_{\varphi}^{-1}\right)^{*}\left(a(\zeta, \theta) e^{i \frac{\pi}{4} \mu}\left|\frac{D\left(\lambda_{1}, \ldots, \lambda_{n}, \varphi_{\theta_{1}}^{\prime}, \ldots, \varphi_{\theta_{N}}^{\prime}\right)}{D\left(\zeta_{1}, \ldots, \zeta_{n}, \theta_{1}, \ldots, \theta_{N}\right)}\right|^{-\frac{1}{2}}\left|d \lambda_{1} \ldots, d \lambda_{n}\right|^{\frac{1}{2}}\right)
$$

where $\lambda_{1}, \ldots, \lambda_{n}$ are functions, homogeneous of degree 1 , inducing coordinates on $C_{\varphi}$ and $\mu$ is an integer, determined by a choice of transversal Lagrangian to $G$ and the fibres, which fixes the Maslov factor.

Hörmander shows that $\sigma_{m}$ is well-defined and extends by linearity to fix (12.1). To get an explicit form for the symbol as in (12.2), observe that there is no necessity to take the functions $\lambda, \ldots, \lambda_{n}$ to be homogeneous of degree 1 . If $n-1$ of them are homogeneous of degree 0 and $\lambda_{n}$ is non-vanishing and homogeneous of degree 
1 the same formula (12.5) holds. In fact $\lambda_{n}$ can be taken to be one of the $\theta$ variables, say $\theta_{N}$ after renumbering. Setting $\varphi(z, \theta)=\theta_{N} \psi\left(z, \theta_{1} / \theta_{N}, \ldots, \theta_{N-1} / \theta_{N}\right)$ and $u_{j}=\theta_{j} / \theta_{N}$ from $j=1, \ldots, N-1$ it follows that (12.5) can be written

$$
\begin{gathered}
\sigma_{m}(u)=\left(p_{\varphi}^{-1}\right)^{*}\left(e^{i \frac{\pi}{4} \mu} a\left|\frac{D\left(\lambda_{1}, \ldots, \lambda_{n-1}, \theta_{N}, \psi_{u_{1}}^{\prime}, \ldots, \psi_{u_{N-1}}^{\prime}, \psi-u \cdot \psi_{u}^{\prime}\right)}{D\left(\zeta_{1}, \ldots, \zeta_{n}, u_{1}, \ldots, u_{N-1}, \theta_{N}\right)}\right|^{-\frac{1}{2}}\right. \\
=\left(p_{\varphi}^{-1}\right)^{*}\left(e^{i \frac{\pi}{4} \mu} a \theta_{N}^{\frac{N}{2}}\left|\frac{D\left(\lambda_{1}, \ldots, \lambda_{n-1}, \psi_{u_{1}}^{\prime}, \ldots, \psi_{u_{N-1}}^{\prime}, \psi\right)}{D\left(\zeta_{1}, \ldots, \zeta_{n}, u_{1}, \ldots, u_{N-1}\right)}\right|^{-\frac{1}{2}}\left|d \lambda_{1} \ldots d \lambda_{N-1} d \theta_{N}\right|^{\frac{1}{2}}\right)
\end{gathered}
$$

In this form we can easily get an explicit version of the symbol for Legendre distributions defined through the Legendre transformation $L$. This gives an isomorphism of bundles, locally,

$$
L^{*}: \mathcal{C}^{\infty}\left(L G ; E^{m}(L G)\right) \longrightarrow \mathcal{C}^{\infty}\left(G ; E^{m}(G)\right)
$$

where on the left $E^{m}$ is the corresponding bundle over the Legendre submanifold G

$$
E^{m}(G)=\left|N^{*} \partial \bar{V}\right|^{m-\frac{n}{4}} \otimes \Omega_{G}^{\frac{1}{2}} \otimes M_{G}
$$

Thus, near $p \in G$, if $\mathbb{S}_{+}^{n} \supset U \stackrel{f}{\longrightarrow} U^{\prime} \subset X$ is a local identification of a neighbourhood of $\pi(p) \in U^{\prime}$ with an open subset of $\mathbb{S}_{+}^{n}$ then

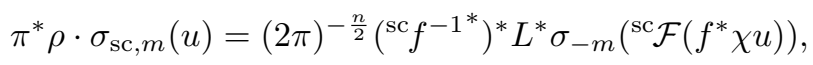

where $\chi \in \mathcal{C}_{c}^{\infty}\left(U^{\prime}\right)$ and ${ }^{\mathrm{sc}} f^{-1^{*}}$ is the lift of $f^{-1}$ to ${ }^{\mathrm{sc}} T^{*} U^{\prime}$ (see (11.4)), defines

$$
\sigma_{\mathrm{sc}, m}(u) \in \mathcal{C}^{\infty}\left(G ; E^{m}(G)\right),
$$

and does so unambiguously. For a particular choice of a defining function of $\partial X$ we will denote a section of $E^{m}(G)$ by $a|d x|^{m-\frac{n}{4}}$ where $a$ is a section of $\Omega_{G}^{\frac{1}{2}} \otimes M_{G}$.

Proposition 12. The symbol map, given locally by (12.9), is well-defined as in (12.10), and gives a short exact sequence

$$
0 \hookrightarrow I_{s c}^{m+1}\left(X, G ;{ }^{s c} \Omega^{\frac{1}{2}}\right) \longrightarrow I_{s c}^{m}\left(X, G ;{ }^{s c} \Omega^{\frac{1}{2}}\right) \longrightarrow \mathcal{C}^{\infty}\left(G ; E^{m}\right) \longrightarrow 0 ;
$$

if $u \in I_{s c}^{m}\left(X, G ;{ }^{s c} \Omega^{\frac{1}{2}}\right)$ is given locally by (11.1) for a parametrization of $G$ then

$$
\begin{aligned}
& \sigma_{s c, m}(u)= \\
& a(0, y, u) e^{i \frac{\pi}{4} \mu}\left|\frac{D\left(\lambda_{1}, \ldots, \lambda_{n-1}, \partial_{u} \varphi\right)}{D\left(y_{1}, \ldots, y_{n-1}, u_{1}, \ldots, u_{k}\right)}\right|^{-\frac{1}{2}}\left|d \lambda_{1}, \ldots, d \lambda_{n-1}\right|^{\frac{1}{2}} \otimes|d x|^{m-\frac{n}{4}}
\end{aligned}
$$

where the $\lambda_{j}$ are coordinate functions on $\left\{\partial_{u} \varphi=0\right\}$.

Using the Fourier transform, as in Proposition 8, to reduce scattering pseudodifferential operators to pseudodifferential operators in the usual sense we can deduce the main symbolic properties of Legendre distributions. 
Proposition 13. Suppose $P \in \Psi_{s c}^{l, k}\left(X ;{ }^{s c} \Omega^{\frac{1}{2}}\right)$ has symbol $x^{k} p_{k}+x^{k+1} p_{k+1}$, as in (10.6) with respect to a product decomposition of $X$ near $\partial X$, and suppose that $G \subset{ }^{s c} T_{\partial X}^{*} X$ is a smooth Legendre submanifold. Then for any $m \in \mathbb{R}$,

$$
\begin{gathered}
P: I_{s c}^{m}\left(X, G ;{ }^{s c} \Omega^{\frac{1}{2}}\right) \longrightarrow I_{s c}^{m+k}\left(X, G ;{ }^{s c} \Omega^{\frac{1}{2}}\right) \\
\sigma_{s c, m+k}(P u)=\left(\left.p_{k}\right|_{G}\right) \sigma_{s c, m}(u) \otimes|d x|^{k} .
\end{gathered}
$$

Furthermore if $p_{k}$ vanishes identically on $G$ then

$$
\begin{gathered}
P: I_{s c}^{m}\left(X, G ;{ }^{s c} \Omega^{\frac{1}{2}}\right) \longrightarrow I_{s c}^{m+k+1}\left(X, G ;{ }^{s c} \Omega^{\frac{1}{2}}\right) \text { and } \\
\sigma_{s c, m+k+1}(P u)= \\
\left(\frac{1}{i}\left(\mathcal{L}_{V}+\left(\frac{1}{2}(k+1)+m-\frac{n}{4}\right) \frac{\partial p_{k}}{\partial \tau}\right)+\left.p_{k+1}\right|_{G}\right) a \otimes|d x|^{m+k+1-\frac{n}{4}}
\end{gathered}
$$

where $\sigma_{s c, m}(u)=a \otimes|d x|^{m-\frac{n}{4}}$ and $V={ }^{s c} H_{p_{k}}^{*, 0}$ is given by (10.7).

Proof. Both these results are sc-microlocal. We shall derive them from the corresponding results in the usual setting; see [3] or [7], Theorem 25.2.4. By assumption, ${ }^{\mathrm{sc}} \mathrm{WF}(u) \Subset{ }^{\mathrm{sc}} T_{\partial X}^{*} X$ so we can assume that $P \in \Psi_{\mathrm{sc}}^{-\infty, k}\left(X,{ }^{\mathrm{sc}} \Omega \frac{1}{2}\right)$. The localization argument in Proposition 6 and the definition of the symbol map (12.7) - (12.10) show that it is only necessary to consider the case of a compactified linear space $\bar{V}$. Now if $v \in I^{-m}\left(V, \Lambda ; \Omega^{\frac{1}{2}}\right) \cap \mathcal{E}^{\prime}\left(V ; \Omega^{\frac{1}{2}}\right)+\mathcal{S}\left(V ; \Omega^{\frac{1}{2}}\right)$ and $Q \in \Psi^{-k}\left(V, \Omega^{\frac{1}{2}}\right)$. then

$$
\begin{gathered}
Q v \in I^{-m-k}\left(V, \Lambda ; \Omega^{\frac{1}{2}}\right) \cap \mathcal{E}^{\prime}\left(V ; \Omega^{\frac{1}{2}}\right)+\mathcal{S}\left(V ; \Omega^{\frac{1}{2}}\right), \\
\sigma_{-m-k}(Q v)=\sigma_{-k}(Q) \sigma_{-m}(v) .
\end{gathered}
$$

Moreover, if $\left.\sigma_{-k}(Q)\right|_{\Lambda}=0$ then

$$
\begin{gathered}
Q v \in I^{-m-k-1}\left(V, \Lambda ; \Omega^{\frac{1}{2}}\right) \cap \mathcal{E}^{\prime}\left(V ; \Omega^{\frac{1}{2}}\right)+\mathcal{S}\left(V ; \Omega^{\frac{1}{2}}\right) \text { and } \\
\sigma_{-m-k-1}(Q v)=\left(\frac{1}{i} \mathcal{L}_{H_{q}}+\left.q\right|_{\Lambda}\right) \sigma_{-m}(v),
\end{gathered}
$$

where $q$ is the invariantly defined Weyl symbol of $Q$ modulo $S^{-k-2}\left(T^{*} V\right)$. In particular, on $\Lambda, q$ is equal to the subprincipal symbol of $Q$. Here we have used the standard notation as in (12.1). To translate this to the form (12.2), consider $Q \in \Psi_{\mathrm{sc}}^{-k, \infty}\left(\bar{V}, \Omega^{\frac{1}{2}}\right)$ with symbol $q$ defined modulo $\rho_{\sigma}^{k+2} \rho_{\partial}^{\infty} \mathcal{C}^{\infty}\left({ }^{\mathrm{sc}} \bar{T}^{*} \bar{V}\right)$. Then (12.15) becomes

$$
\sigma_{-k-m}^{\sharp}(Q v)=\left(\left.\rho_{\sigma}^{-k} q\right|_{\Lambda \cap S^{*} V}\right) \cdot \sigma_{-m}^{\sharp}(v) \otimes\left|d \rho_{\sigma}\right|^{k} .
$$

If $u \in I_{\mathrm{sc}}^{m}\left(\bar{V} ; G,{ }^{\mathrm{sc}} \Omega^{\frac{1}{2}}\right)$ then by Proposition 10 ,

$$
v={ }^{\mathrm{sc}} \mathcal{F} u \in I^{-m}\left(V, \Lambda, \Omega^{\frac{1}{2}}\right) \cap \mathcal{E}^{\prime}\left(V, \Omega^{\frac{1}{2}}\right)+\mathcal{S}\left(V, \Omega^{\frac{1}{2}}\right),
$$

where $\Lambda \cap S^{*} V=L(G)$ with $L$ defined in Lemma 5. If $P \in \Psi_{\mathrm{sc}}^{-\infty, k}\left(\bar{V},{ }^{\mathrm{sc}} \Omega^{\frac{1}{2}}\right)$ then, by Proposition $8, Q={ }^{\mathrm{sc}} \mathcal{F} \circ P \circ{ }^{\mathrm{sc}} \mathcal{F}^{-1} \in \Psi^{-k, \infty}\left(\bar{V}\right.$, $\left.{ }^{\mathrm{sc}} \Omega^{\frac{1}{2}}\right)$ and the symbol, $p$, of $P$, is related to the symbol, $q$, of $Q$ by $p=\widetilde{L}^{*} q$ where $p$ and $q$ are invariantly defined modulo $\rho_{\partial}^{k+2} \rho_{\sigma}^{\infty} \mathcal{C}^{\infty}$ and $\rho_{\sigma}^{k+2} \rho_{\partial}^{\infty} \mathcal{C}^{\infty}$ respectively. Hence from the definitions (12.9) and (12.17)

$$
\begin{gathered}
\sigma_{k+m}(P u)=L^{*} \sigma_{-k-m}(Q v)= \\
L^{*}\left(\left.\rho_{\sigma}^{-k} q\right|_{\Lambda \cap S^{*} V}\right) L^{*} \sigma_{-m}^{\sharp}(v) \otimes L^{*}\left|d \rho_{\sigma}\right|^{k}=\left(\left.p_{k}\right|_{G}\right) \sigma_{\mathrm{sc}, m}(u) \otimes|d x|^{k}
\end{gathered}
$$


which is (12.13).

To obtain (12.14) we keep the same notation and note that $\left.p_{k}\right|_{G} \equiv 0$ is equivalent to $\left.\rho_{\sigma}^{-k} q\right|_{\Lambda \cap S^{*} V} \equiv 0$. That is $\left.\sigma_{-k}(Q)\right|_{\Lambda} \equiv 0$. From (12.16) we get, by identifying $\sigma_{m}(A)$ with $\rho_{\sigma}^{-m} \sigma^{\sharp}(A)$

$$
\sigma_{-k-m-1}^{\sharp}(Q v)=\left(\frac{1}{i} \mathcal{L}_{H_{q}}+\left.q\right|_{\Lambda}\right)\left(\sigma_{-m}^{\sharp}(v) \rho_{\sigma}^{m}\right) .
$$

Now,

$$
\begin{aligned}
\frac{1}{i} \mathcal{L}_{H_{q}}+\left.q\right|_{\Lambda} & =\frac{1}{i} \mathcal{L}_{\rho_{\sigma}^{k+1} W}+\left.\rho_{\sigma}^{k+1}\left(q / \rho_{\sigma}^{k+1}\right)\right|_{\Lambda \cap S^{*} V} \\
& =\rho_{\sigma}^{k+1}\left(\frac{1}{i}\left(\mathcal{L}_{W}+\frac{1}{2}(k+1) \rho_{\sigma}^{-1} W \rho_{\sigma}\right)+q /\left.\rho_{\sigma}^{k+1}\right|_{\Lambda \cap S^{*} V}\right)
\end{aligned}
$$

where the reduced Hamilton vector field $W={ }^{\mathrm{sc}} H_{q}^{-k,-1}$ is tangent to $S^{*} V=\left\{\rho_{\sigma}=\right.$ $0\}$ with the choice of the $\rho_{\partial}$ scaling, -1 , irrelevant. In the formula above we have again identified a homogeneous function, $f$, on $\Lambda$ with $\rho_{\sigma}^{m} f^{\sharp}$, where $f^{\sharp}$ is a function on $\Lambda \cap S^{*} V$. The second equality follows from the general formula for the conformal transformation of the Lie derivative of a vector field on half-densities:

$$
\mathcal{L}_{f Z}=f \mathcal{L}_{Z}+\frac{1}{2} Z f
$$

Hence

(12.19) $\sigma_{-k-m-1}^{\sharp}(Q v)=$

$$
\left(\frac{1}{i}\left(\mathcal{L}_{W}+\left.\frac{1}{2}(k+1) \rho_{\sigma}^{-1} W \rho_{\sigma}\right|_{\Lambda \cap S^{*} V}\right)+q /\left.\rho_{\sigma}^{k+1}\right|_{\Lambda \cap S^{*} V}\right) \sigma_{-m}^{\sharp}(v) \otimes|d \rho|^{k+1} .
$$

If $\sigma_{-m}^{\sharp}(v)=b\left|d \rho_{\sigma}\right|^{m-n / 4}$ then the application of the Lie derivative gives

$$
\begin{aligned}
\sigma_{-k-m-1}^{\sharp}(Q v)=\left(\frac { 1 } { i } \left(\mathcal{L}_{W} b+\left(\frac{1}{2}(\right.\right.\right. & \left.\left.k+1)+m-\frac{n}{4}\right) \rho_{\sigma}^{-1} W \rho_{\sigma}\right) \\
& \left.+q /\left.\rho_{\sigma}^{k+1}\right|_{S^{*} V \cap \Lambda}\right) b \otimes\left|d \rho_{\sigma}\right|^{m+k+1-\frac{n}{4}}
\end{aligned}
$$

This gives (12.14) since $\tilde{L}_{*} V=W, \sigma_{\mathrm{sc}, k+m+1}(v)=L^{*} \sigma_{-k-m-1}^{\sharp}(Q v), \sigma_{\mathrm{sc}, m}(u)=$ $a\left|d \rho_{\partial}\right|^{m-n / 4}, a=L^{*} b$ and $\left|d \rho_{\partial}\right|=L^{*}\left|d \rho_{\sigma}\right|$ (and of course, $x=\rho_{\partial}$ ).

\section{CONIC POINTS}

In $\S 11$ and $\S 12$ the definition and symbolic properties of Legendre distributions associated to a smooth Legendre submanifold of ${ }^{\mathrm{sc}} T_{\partial X}^{*} X$ are discussed. As noted in $\S 4$ the Legendre variety $\widetilde{G}_{y}(\lambda)$, to which the generalized plane wave with frequency $\lambda$, emanating from $y \in \partial X$, is associated is, in general, an intersecting pair of Legendre submanifolds with conic points. The parametrization of such a Legendre manifold has been described in $\S 7$. We extend the notion of a Legendre distributions, as in Definition 2, from the smooth case by introducing appropriate singular amplitudes. 
Definition 3. For an intersecting pair of Legendre submanifolds with conic points, in the sense of $(5.2)-(5.6)$, and any $m, p \in \mathbb{R}$ the space $I_{s c}^{m, p}\left(X, \widetilde{G} ;{ }^{s c} \Omega^{\frac{1}{2}}\right)$ consists of those $u \in \mathcal{C}^{-\infty}\left(X ;{ }^{s c} \Omega^{\frac{1}{2}}\right)$ of the form

$$
u=u_{0}+u_{+}+\sum_{j=1}^{N} u_{j}
$$

where $u_{0} \in I_{s c}^{m}\left(X, G ;{ }^{s c} \Omega^{\frac{1}{2}}\right), u_{+} \in I_{s c}^{p}\left(X, G^{\sharp} ;{ }^{s c} \Omega^{\frac{1}{2}}\right)$ and the $u_{j}$ are given in terms of local parametrizations, $\varphi_{j}$ of $\bar{G}$ near $\widehat{G}_{0}$, the conic limit, by integrals

$$
\begin{aligned}
& u_{j}=(2 \pi)^{-\frac{n}{4}-\frac{k+1}{2}} \int_{0}^{\infty} \int_{\mathbb{R}^{k}} e^{i \varphi_{j}(y, s, u) / x} a_{j}\left(\frac{x}{s}, y, s, u, x\right) \cdot\left(\frac{x}{s}\right)^{m+\frac{n}{4}-\frac{k+1}{2}} s^{p+\frac{n}{4}-1} d s d u \nu \\
& \text { with } a_{j} \in \mathcal{C}_{c}^{\infty}\left([0, \infty) \times \mathbb{R}^{n-1} \times[0, \infty) \times \mathbb{R}^{k} \times[0, \infty)\right) \text { and } \nu \in \mathcal{C}^{\infty}\left(X ;{ }^{s c} \Omega^{\frac{1}{2}}\right)
\end{aligned}
$$

Notice that, for $x>0$, the integrals in (13.2) are absolutely convergent due to the compactness of the support of the $a_{j}$ in $u, x / s$ and $s$. Thus the integral defines a tempered distributional half-density on $X$, i.e. an element of the dual of $\mathcal{C}^{\dot{\infty}}\left(X,{ }^{{ }^{s c}} \Omega^{\frac{1}{2}}\right)$. If $a_{j}$ is supported in $\{s>0\}$ then $\varphi_{j}$, with parameters $(s, u)$, is a parametrization of $G$, the smooth part of $\bar{G}$, and $u_{j}$ contributes a term to $I_{\mathrm{sc}}^{m}\left(X, G ;{ }^{\mathrm{sc}} \Omega^{\frac{1}{2}}\right)$. A small modification of Lemma 8 shows that if $A \in \Psi_{\mathrm{sc}}^{0,0}\left(X,{ }^{\mathrm{sc}} \Omega^{\frac{1}{2}}\right)$ has essential support away from $\bar{G} \backslash G$ and $u \in I_{\mathrm{sc}}^{m, p}\left(X, \widetilde{G} ;{ }^{\mathrm{sc}} \Omega^{\frac{1}{2}}\right)$ then $A u \in I_{\mathrm{sc}}^{m}\left(X, G ;{ }^{\mathrm{sc}} \Omega^{\frac{1}{2}}\right)$.

Lemma 9. For any $u \in I_{s c}^{m, p}\left(X, \bar{G} ;{ }^{s c} \Omega^{\frac{1}{2}}\right)$,

$$
{ }^{s c} W F(u) \subset \bar{G}
$$

and, given any covering of $\widehat{G}_{0}$ by parametrizations, $u$ can be written in the form (13.1), (13.2) with respect to these parametrizations.

Proof. Since the terms $u_{0}$ and $u_{+}$in (13.1) clearly satisfy (13.3), it is enough to consider an oscillatory integral as in (13.2). Applying $A \in \Psi_{\mathrm{sc}}^{0,0}\left(X ;{ }^{\mathrm{sc}} \Omega^{\frac{1}{2}}\right)$ gives, by Lemma 8, an integral of the same form. Moreover, if $A$ is microlocally of order $\infty$ near $\bar{G}$ then the resulting amplitude $a^{\prime}$, vanishes rapidly as $x \rightarrow 0$ near both the critical points of $\varphi$ and the surface $s=0$ (since the latter corresponds to $G^{\sharp}$ ). Integration by parts therefore shows that $u_{j} \in \dot{\mathcal{C}}^{\infty}\left(X ;{ }^{\mathrm{sc}} \Omega^{\frac{1}{2}}\right)$, so (13.3) holds.

The second part of the lemma follows from the equivalence of phase functions, as given by Proposition 7 , and the use of stationary phase to change the number of $u$ parameters near $s=0$.

The coordinate invariance of these spaces is immediate from the definition. To extend the invariant symbol map to this larger class of distributions it suffices to work locally and use continuity.

Recall that $\widehat{G}=\beta^{*} G \subset\left[{ }^{s c} \bar{T}_{\partial X}^{*} X ; \operatorname{sp}\left\{\frac{d x}{x^{2}}\right\}\right]$ is the blown-up version of $\bar{G}$; it is a smooth manifold with corner corresponding to the introduction of $\mu$-polar coordinates around $\mu=0$, with a natural blow-down map

$$
\beta: \widehat{G} \longrightarrow \bar{G} \text {. }
$$

This is an isomorphism of $\widehat{G} \backslash \widehat{G}_{0}, \widehat{G}_{0}=\partial \widehat{G}$, onto $G$, the regular part of $\bar{G}$. 
As noted in $\S 9$, the Maslov bundle extends naturally from $G$ to $\widehat{G}$. Let $S \in \mathcal{C}^{\infty}(\widehat{G})$ be a defining function for the boundary, $\widehat{G}_{0}$ of $\widehat{G}$. Then for any $a \in \mathbb{R}$, and any bundle $L$,

$$
S^{a} \mathcal{C}^{\infty}(\widehat{G} ; L)=\mathcal{C}^{\infty}\left(\widehat{G} ; S^{a} \otimes L\right)=\mathcal{C}^{\infty}\left(\widehat{G} ;\left|N^{*} \partial\left({ }^{\mathrm{sc}} \widehat{T}_{\partial X}^{*} X\right)\right|_{\widehat{G}}^{a} \otimes L\right)
$$

defines a 'rescaled' bundle $S^{a} \otimes L=\left|N^{*} \partial\left({ }^{\mathrm{sc}} \widehat{T}_{\partial X}^{*} X\right)\right|_{\widehat{G}}^{a} \otimes L$ over $\widehat{G}$. Here we have used the same notation as in the definition of the symbol map in the previous section. We now define the symbol bundle over $\widehat{G}$ to be

$$
\hat{E}^{m, p}(\widehat{G})=\left|N^{*} \partial\left({ }^{\mathrm{sc}} \widehat{T}_{\partial X}^{*} X\right)\right|_{\widehat{G}}^{p-m} \otimes\left|N^{*} \partial X\right|^{m-\frac{n}{4}} \otimes \Omega_{\mathrm{b}}^{\frac{1}{2}}(\widehat{G}) \otimes M_{\widehat{G}}
$$

where $\Omega_{\mathrm{b}}^{\frac{1}{2}}(\widehat{G})$ is the b-half-density bundle of $\widehat{G}$. When specific defining functions of the boundaries are chosen $\left(x, S\right.$, trivializing $\left.S^{a}\right)$ we will write a section as $u=$ $a|d S|^{p-m}|d x|^{m-\frac{n}{4}}, a \in \mathcal{C}^{\infty}\left(\widehat{G} ; \Omega_{\mathrm{b}}^{\frac{1}{2}} \otimes M_{\widehat{G}}\right)$.

Proposition 14. The symbol map (12.11) extends by continuity to define

$$
\hat{\sigma}_{s c, m, p}: I_{s c}^{m, p}\left(X, \widetilde{G} ;{ }^{s c} \Omega^{\frac{1}{2}}\right) \longrightarrow \mathcal{C}^{\infty}\left(\widehat{G} ; \hat{E}^{m, p}\right)
$$

which gives a short exact sequence

$$
0 \longrightarrow I_{s c}^{m+1, p}\left(X, \widetilde{G} ;{ }^{s c} \Omega^{\frac{1}{2}}\right) \longrightarrow I_{s c}^{m, p}\left(X, \widetilde{G} ;{ }^{s c} \Omega^{\frac{1}{2}}\right) \longrightarrow \mathcal{C}^{\infty}\left(\widehat{G} ; \hat{E}^{m, p}\right) \longrightarrow 0 .
$$

Proof. Consider a half-density of the form (13.2). Microlocally near $G$ this is a Legendre distribution in the sense of $\S 11$. Its symbol is fixed by (12.5). The Lagrangian distribution $F G^{*} u$ is of the form (11.10), and (11.11), with only different notation for the parameters. Thus $\hat{\sigma}_{\mathrm{sc}, m, p}$ is defined by continuity. The exactness in (13.8) follows from Lemma 9 and integration by parts.

Proposition 15. Under the hypotheses of Proposition 13, if $G$ is the smooth part of an intersecting pair of Legendre submanifolds with conic points then

$$
\begin{gathered}
P: I_{s c}^{m, p}\left(X, \widetilde{G} ;{ }^{s c} \Omega^{\frac{1}{2}}\right) \longrightarrow I_{s c}^{m+k, p+k}\left(X, \widetilde{G} ;{ }^{s c} \Omega^{\frac{1}{2}}\right) \\
\text { with } \hat{\sigma}_{s c, m+k, p+k}(P u)=\beta^{*}\left(\left.p_{k}\right|_{\bar{G}}\right) \cdot \hat{\sigma}_{s c, m, p}(u)
\end{gathered}
$$

and if $p_{k}=0$ on $\widetilde{G}$ then

$$
P: I_{s c}^{m, p}\left(X, \widetilde{G} ;{ }^{s c} \Omega^{\frac{1}{2}}\right) \longrightarrow I_{s c}^{m+k+1, p+k+1}\left(X, \widetilde{G} ;{ }^{s c} \Omega^{\frac{1}{2}}\right)
$$

and formula (12.14) extends by continuity to

$$
\begin{aligned}
\sigma_{s c, m+k+1, p+k+1} & (P u) \\
=\left(\frac{1}{i}\right. & \left(\mathcal{L}_{V}+\left(\frac{1}{2}(k+1)+m-\frac{n}{4}\right) \beta^{*} \frac{\partial p_{k}}{\partial \tau}+(p-m) S^{-1} V S\right) \\
& \left.\quad+\beta^{*}\left(\left.p_{k+1}\right|_{G}\right)\right) a \otimes|d x|^{m+k+1-\frac{n}{4}} \otimes|d S|^{p-m}
\end{aligned}
$$

where $\sigma_{s c, m, p}(u)=a \otimes|d x|^{m-\frac{n}{4}} \otimes|d S|^{p-m}$ and $V \in \mathcal{V}_{b}(\widehat{G})$ is determined by the requirement $\beta_{*} V={ }^{s c} H_{p}^{m, p}$.

Proof. Let $U$ and $U_{1}$ be open subsets of ${ }^{\text {sc }} \bar{T}^{*}{ }_{\partial X} X$ such that $\bar{U} \Subset U_{1}$ and $\bar{G} \cap U_{1} \Subset G$. Then we can find $A \in \Psi_{\mathrm{sc}}^{0,0}\left(X,{ }^{\mathrm{sc}} \Omega^{\frac{1}{2}}\right)$ such that $u_{1}=A u$ satisfies ${ }^{\mathrm{sc}} \mathrm{WF}\left(u-u_{1}\right) \subset$ ${ }^{\mathrm{sc}} \bar{T}^{*}{ }_{\partial X} X \backslash U,{ }^{\mathrm{sc}} \mathrm{WF}\left(u_{1}\right) \subset U_{1}$; see the remarks following Definition 2 . Hence, $u_{1} \in$ 
$I_{\mathrm{sc}}^{m}\left(X, G ;{ }^{\mathrm{sc}} \Omega^{\frac{1}{2}}\right)$ and since $\hat{E}^{m, p}(\widehat{G})$ can be identified with $\beta^{*} E^{m}(G)$ over compact sets in the interior of $\widehat{G}$ we get

$$
\left.\hat{\sigma}_{\mathrm{sc}, m, p}(u)\right|_{\beta^{*} U}=\beta^{*}\left(\left.\sigma_{\mathrm{sc}, m}\left(u_{1}\right)\right|_{U}\right) .
$$

Thus using (12.13) we obtain

$$
\left.\hat{\sigma}_{\mathrm{sc}, m+k, p+k}(P u)\right|_{\beta^{*} U}=\left.\left(\beta^{*}\left(\left.p_{k}\right|_{G}\right) \hat{\sigma}_{\mathrm{sc}, m, p}(u)\right)\right|_{\beta^{*} U} .
$$

By expanding the open sets $U$ we can replace $\beta^{-1}(U)$ by $\widehat{G} \backslash \partial \widehat{G}$ and then the continuity of both sides gives (13.9). We obtain (13.11) similarly from (12.14).

We also note the structure of the residual spaces for the symbol filtration (13.8):

$$
I_{\mathrm{sc}}^{\infty, p}\left(X, \widetilde{G} ;{ }^{\mathrm{sc}} \Omega^{\frac{1}{2}}\right)=\bigcap_{m} I_{\mathrm{sc}}^{m, p}\left(X, \widetilde{G} ;{ }^{\mathrm{sc}} \Omega^{\frac{1}{2}}\right)=I_{\mathrm{sc}}^{p}\left(X, G^{\sharp} ;{ }^{\mathrm{sc}} \Omega^{\frac{1}{2}}\right) .
$$

Iterative use of (13.8) will allow us to construct distributions modulo errors which are oscillatory functions associated to $G^{\sharp}$. For use in $\S 15$ we also note the asymptotic completeness of these spaces.

Lemma 10. Let $\widetilde{G}$ be an intersecting pair of Legendre submanifolds with conic points and suppose $u_{j} \in I_{s c}^{m+j, p}\left(X, \widetilde{G} ;{ }^{s c} \Omega^{\frac{1}{2}}\right)$, for $j \in \mathbb{N}_{0}$. Then there exists $u \in$ $I_{s c}^{m, p}\left(X, \widetilde{G},{ }^{s c} \Omega^{\frac{1}{2}}\right)$ such that for any $N \in \mathbb{N}$

$$
u-\sum_{j=0}^{N} u_{j} \in I_{s c}^{m+N+1, p}\left(X, \widetilde{G},{ }^{s c} \Omega^{\frac{1}{2}}\right) .
$$

Proof. This follows as in the standard case by selecting a covering of the Legendre variety by phase functions and decomposing each of the $u_{j}$ into a sum of oscillatory integrals with respect to these phase functions using Lemma 9. Then the standard Borel procedure can be used to asymptotically sum the series of amplitudes.

As already noted a smooth Legendre manifold may also be considered as a conic Legendre variety. Thus, suppose $G \subset{ }^{\mathrm{sc}} T_{\partial X}^{*} X$ is smooth and that for some boundary defining function $x$ and $\lambda \in \mathbb{R}$,

$$
\begin{gathered}
G \cap G^{\sharp}(\lambda)=Y \text { is a } \mathcal{C}^{\infty} \text { submanifold around which } \\
G \text { is conic in the sense of (5.10). }
\end{gathered}
$$

Notice that $\tau$ is constant on $Y$ which therefore projects diffeomorphically to a submanifold of $\partial X$, which we also denote simply as $Y$.

Lemma 11. If $G$ is a smooth Legendre manifold satisfying (13.13) and $Y$ has codimension $L$ in $\partial X$ then

$$
I_{s c}^{m}\left(X, G ;{ }^{s c} \Omega^{\frac{1}{2}}\right) \subset I_{s c}^{m, m+\frac{1}{2} L}\left(X, \widetilde{G} ;{ }^{s c} \Omega^{\frac{1}{2}}\right)
$$

where $\widetilde{G}=G \sqcup G^{\sharp}(\lambda)$.

Proof. It suffices to consider $u \in I_{\mathrm{sc}}^{m}\left(X, G\right.$; $\left.{ }^{\text {sc }} \Omega^{\frac{1}{2}}\right)$ with support near some point of $Y$. Thus $u$ can be taken to be of the form (11.1) for a given parametrization of $G$. In appropriate coordinates $x, y^{\prime}, y^{\prime \prime}, \mu^{\prime}, \mu^{\prime \prime}, \tau$ in which $Y=\left\{y^{\prime}=0\right\}, G$ must be defined by (6.9) where $\mu^{\prime}=0$ implies that $\tau$ is constant, $y^{\prime}=0$ and $\mu^{\prime \prime}=0$. This gives a parametrization (6.10) where $\phi\left(y, \mu^{\prime}\right)=-\lambda+\psi\left(y, \mu^{\prime}\right)$ and $\psi(y, 0)=0$. It follows that the introduction of polar coordinates in the parameters, $\mu^{\prime}=s u$, 
$u=\mu^{\prime} /\left|\mu^{\prime}\right|$ gives a conic parametrization of $\widetilde{G}$. Inserting this change of variable in (11.1) reduces it to the form (13.2) with $k=L-1$ and $p=m+\frac{1}{2} L$.

\section{Push-Forward}

The elements of $I_{\mathrm{sc}}^{m, p}\left(X, \widetilde{G}\right.$; $\left.{ }^{\mathrm{sc}} \Omega^{\frac{1}{2}}\right)$ have distributional asymptotic expansions as $x \downarrow 0$; this indeed is how the scattering matrix arises. For any product decomposition near the boundary we define the partial pairing

$$
\begin{gathered}
B: \mathcal{C}^{-\infty}\left(X ;{ }^{\mathrm{sc}} \Omega^{\frac{1}{2}}\right) \times \mathcal{C}^{\infty}\left(\partial X ; \Omega^{\frac{1}{2}}\right) \longrightarrow \mathcal{C}^{-\infty}\left([0, \epsilon),{ }^{\mathrm{sc}} \Omega^{\frac{1}{2}}\right) \\
\text { by } B(u, f)=x^{\frac{n-1}{2}} \int_{\partial X} u(x, y) f(y) .
\end{gathered}
$$

The symbolic map properties of this pairing can be described in terms of the following map.

Lemma 12. The isomorphism $\gamma$ in (5.11) defines a natural isomorphism between sections of line bundles

$$
\begin{aligned}
& \gamma^{*}: \mathcal{C}^{\infty}\left(S^{*} \partial X ;\left|N^{*} \partial \bar{T}^{*} X\right|^{a}\right) \longrightarrow \\
&\left.\mathcal{C}^{\infty}\left(\partial^{s c} \widehat{T}_{\partial X}^{*} X\right) ;\left|N^{*} \partial\left({ }^{s c} \widehat{T}_{\partial X}^{*} X\right)\right|^{-a} \otimes\left|N^{*} \partial X\right|^{a}\right)
\end{aligned}
$$

Proof. If $(x, y ; \tau, \mu)$ are canonical coordinates in ${ }^{\mathrm{sc}} T^{*} X$, such that

$$
G^{\sharp}=\bar{\tau} \operatorname{graph}\left\{d x / x^{2}\right\},
$$

then $(y, \tilde{\mu}) \mapsto(0, y ; \bar{\tau}, \tilde{\mu})$ are coordinates on the front face of the blown-up manifold $\left(\beta^{*} \operatorname{sp}\left(d x / x^{2}\right)\right)^{\circ}=\left(\mathrm{ff}^{\mathrm{s}} \widehat{T}^{*} X\right)$, where $\tilde{\mu}=\mu / x$. In these coordinates $\gamma$ is the identity in the interior. Coordinates on $\bar{T}^{*} \partial X$ near $\partial \bar{T}^{*} \partial X$ are $\left(y ;|\tilde{\mu}|^{-1}, \tilde{\mu} /|\tilde{\mu}|\right)$, that is, formally $(y ; x /|\mu|, \hat{\mu})$. A section of $\mathcal{C}^{\infty}\left(S^{*} \partial X ;\left|N^{*} \partial \bar{T}^{*} \partial X\right|^{a}\right)$ can therefore be identified with $(x /|\mu|)^{a} \phi(y, \hat{\mu})$, where $\phi \in \mathcal{C}^{\infty}\left(S^{*} \partial X\right)$. On the other hand the coordinates on ${ }^{\mathrm{sc}} \widehat{T}_{\partial X}^{*} X$ are given by $(y, \tau,|\mu|, \hat{\mu}) \mapsto(0, y ; \tau,|\mu|, \hat{\mu}), \mu=|\mu| \hat{\mu}$, and thus on $\partial\left({ }^{\mathrm{s} c} \widehat{T}_{\partial X}^{*} X\right)$ by $(y, \tau, \hat{\mu}) \mapsto(0, y ; \tau, 0, \hat{\mu})$. It follows that an element of the right hand side of $(14.2)$ can be written as $(x /|\mu|)^{a} \psi(y, \hat{\mu})$ and hence it is identified with an element of the left hand side by $\gamma^{*}$.

Using (14.2) we can now state

Proposition 16. For any intersecting pair of Legendre submanifolds with conic points $G$, the partial pairing (14.1) gives a map

$$
B: I_{s c}^{m, p}\left(X, \widetilde{G} ;{ }^{s c} \Omega^{\frac{1}{2}}\right) \times \mathcal{C}^{\infty}\left(\partial X ; \Omega^{\frac{1}{2}}\right) \longmapsto \sum_{j} I_{s c}^{p+\frac{n-1}{4}}\left([0, \epsilon), G^{\prime}\left(\bar{\tau}_{j}\right) ;{ }^{s c} \Omega^{\frac{1}{2}}\right)
$$

where the $G^{\prime}\left(\bar{\tau}_{j}\right)=\left\{\left(0,-\bar{\tau}_{j} d x / x^{2}\right)\right\} \subset{ }^{s c} T_{0}^{*}[0, \epsilon)$ are the Legendre submanifolds (in this case points) corresponding to the components of $G^{\sharp}$ and (14.3) can be written explicitly as

$$
B(u, f)=\sum_{j} e^{-i \bar{\tau} / x} x^{p+\frac{n}{4}} Q_{\bar{\tau}_{j}}(u, f)\left|\frac{d x}{x^{2}}\right|^{\frac{1}{2}}
$$

with $Q_{\bar{\tau}}(u, f) \in \mathcal{C}^{\infty}([0, \epsilon))$, for each $\bar{\tau}=\bar{\tau}_{j}$, satisfying

$$
\left.Q_{\bar{\tau}}(u, f)\right|_{x=0}=\left\langle Q_{\bar{\tau}}^{0}(u), f\right\rangle,
$$


where

$$
\begin{gathered}
Q_{\bar{\tau}}^{0}(u) \in I^{p-m-\frac{n-1}{4}}\left(\partial X, \Lambda(\widetilde{G} ; \bar{\tau}) ; \Omega^{\frac{1}{2}}\right), \\
\gamma^{*}\left(\sigma_{p-m-\frac{n-1}{4}}^{\sharp}\left(Q_{\bar{\tau}}^{0}\right)\right)=\left.\hat{\sigma}_{\mathrm{sc}, m, p}(u)\right|_{\widehat{G}_{0} \cap \beta^{*} G^{\sharp}(\bar{\tau})} \otimes|d x|^{-p+\frac{n}{4}},
\end{gathered}
$$

the Lagrangian $\Lambda(\widetilde{G}, \bar{\tau})$ being given by Proposition 4 .

Proof. We can use the local formula (13.2) once we observe that $u_{0}$ and $u_{+}$in (13.1) do not contribute to the singularities of $Q_{\bar{\tau}}^{0}(u)$. In fact, the assumption ${ }^{\mathrm{sc}} \mathrm{WF}\left(u_{0}\right) \cap$ $\{\mu=0\}=\emptyset$ (implied by (5.4)) shows that in a local representation (11.1) of $u_{0}$, $\phi_{y}^{\prime} \neq 0$ on the support of $a$. Hence there are no stationary points in the oscillatory integral

$$
(2 \pi)^{-\frac{n}{4}-\frac{k}{2}} \int e^{i \phi(y, u) / x} a(x, y, u) f(y) x^{m-\frac{k}{2}+\frac{n}{4}} d u d y .
$$

It follows that this term is $\mathcal{C}^{\infty}$ in $x$ down to $x=0$ where it vanishes to infinite order. The term $u_{+}$is already locally of the form $\exp (-i \bar{\tau} / x) x^{p+\frac{n}{4}} a(x, y)$, with $a \in \mathcal{C}^{\infty}(X)$ and hence $(14.3)-(14.5)$ hold for it with $Q_{\bar{\tau}}^{0}(y)=a(0, y) \in \mathcal{C}^{\infty}(\partial X)$. Thus the only non-trivial contributions to the singularities of $Q_{\bar{\tau}}^{0}(u)$ come from the terms of the form (13.2). These can be rewritten

$$
\begin{aligned}
e^{-i \bar{\tau} / x} x^{p+\frac{n}{4}}(2 \pi)^{-\frac{n}{4}-\frac{k+1}{2}} & \int_{0}^{\infty} \int_{\mathbb{R}^{k}} e^{i \frac{s}{x} \psi_{1}(y, u)+i\left(\frac{s}{x}\right)^{2} x \psi_{2}(y, u, s)} \\
& a\left(\frac{x}{s}, y, s, u, x\right)\left(\frac{s}{x}\right)^{p-m+\frac{k+1}{2}} d u d\left(\frac{s}{x}\right)\left|\frac{d y}{x^{n-1}}\right|^{\frac{1}{2}}\left|\frac{d x}{x^{2}}\right|^{\frac{1}{2}},
\end{aligned}
$$

where $\phi(y, u, s)=-\bar{\tau}+s \psi(y,, u, s)$ and $\psi(y, u, s)=\psi_{1}(y, u)+s \psi_{2}(y, u, s)$.

Changing variables to $\eta=s / x$ we obtain

$$
\begin{aligned}
e^{-i \bar{\tau} / x} x^{p+\frac{n}{4}}(2 \pi)^{-\frac{n}{4}-\frac{k+1}{2}} & \int_{0}^{\infty} \int_{\mathbb{R}^{k}} e^{i \eta \psi_{1}(y, u)+i \eta^{2} x \psi_{2}(y, u, x \eta)} \\
& a\left(\frac{1}{\eta}, y, u, x\right) \eta^{p-m+\frac{k+1}{2}} d u d \eta\left|\frac{d y}{x^{n-1}}\right|^{\frac{1}{2}}\left|\frac{d x}{x^{2}}\right|^{\frac{1}{2}} .
\end{aligned}
$$

When pairing with $f \in \mathcal{C}^{\infty}(\partial X)$ according to (14.1) the orders of integration can be freely interchanged for $x>0$. It can also be assumed that the support in $s$ in the original integrand is arbitrarily close to 0 , since the contribution from $s>\epsilon$ can be absorbed in the term $u_{0}$. Hence $x \eta$ is small in the integrand and consequently the nonvanishing of $d_{(y, u)} \psi_{1}$ shows that the result of integration in $y$ and $u$ decays rapidly in $\eta$ independently of $x \geq 0$ with all derivatives in $x$ decaying as well. This gives $(14.3)-(14.5)$ with

$$
Q_{\bar{\tau}}^{0}(u)=(2 \pi)^{-\frac{n}{4}-\frac{k+1}{2}} \int_{0}^{\infty} \int_{\mathbb{R}^{k}} e^{i \eta \psi_{1}(y, u)} a\left(\frac{1}{\eta}, y, u, 0\right) \eta^{p-m+\frac{k+1}{2}} d u d \eta|d y|^{\frac{1}{2}}
$$

As in (7.7) we put $\mathbb{R}^{k+1} \ni \theta=\left(\theta_{1}, \theta^{\prime}\right)=\eta(1, u)$ and define $\phi(y, \theta)$ in a conic neighbourhood of $(1,0)$ as $\theta_{1} \psi_{1}\left(y, \theta^{\prime} / \theta_{1}\right)$. This parametrizes $\Lambda(\widetilde{G}, \bar{\tau})$ so from the standard order convention $Q_{\bar{\tau}}^{0}(u) \in I^{p-m-\frac{n-1}{4}}\left(\partial X, \Lambda(\widetilde{G}, \bar{\tau}) ; \Omega^{\frac{1}{2}}\right)$. The natural symbol identification (14.6) follows from (14.9) and the discussion of the symbol map in $\S 12$. 
Note that the partial pairing (14.1) and the definition of $Q_{\bar{\tau}}^{0},(14.4)$ and (14.5) depend on the choice of the defining function. At the symbol level this dependence is precisely described by (14.6).

The proof of Proposition 16 gives in fact a little more and the following will be useful in the construction of the Poisson kernel:

Lemma 13. In the notation of Proposition 16

$$
\begin{gathered}
\left.\left(\frac{d}{d x}\right)^{l} Q_{\bar{\tau}}(u, f)\right|_{x=0}=\left\langle Q_{\bar{\tau}}^{l}(u), f\right\rangle \\
\text { where } Q_{\bar{\tau}}^{l}(u) \in I^{p-m-\frac{n-1}{4}+2 l}\left(\partial X, \Lambda(\widetilde{G}, \bar{\tau}) ; \Omega^{\frac{1}{2}}\right) .
\end{gathered}
$$

As shown by Lemma 11, Proposition 16 can be applied in the case of a smooth Legendre manifold satisfying (13.13). The lemma of stationary phase gives a more precise description of the push-forward.

Proposition 17. If $G$ is a smooth Legendre variety satisfying (13.13) and $u \in$ $I_{s c}^{m}\left(X, G ;{ }^{s c} \Omega^{\frac{1}{2}}\right)$ then the distributions $Q_{\bar{\tau}}^{l}(u)$ defined using (13.14) and (14.5) are all differentiated Dirac distributions on $Y$, i.e. conormal distributions supported on $Y$, and $Q_{\bar{\tau}}^{0}(u)$ is a Dirac delta distribution.

Proof. This follows by a direct stationary phase argument, but can also be deduced from Proposition 16. Indeed, the absence of any terms associated to the Legendre manifold $G^{\sharp}(\lambda)$ means, following the proof of that proposition, that the $Q_{\bar{\tau}}^{l}(u)$ are supported on $Y$. The order of these distributions is $L / 2+(n-1) / 4$, where $L$ is the codimension of $Y$ and this implies the last statement, since the Delta distributions are the only conormal distributions supported on the submanifold and of this order.

\section{Parametrix COnstruction}

The Poisson operator $P(\lambda)$ is defined by (0.4). In this section the symbol calculus developed above is used to construct a parametrix for $P(\lambda)$. The kernel of the parametrix is a Legendre distribution on the manifold with boundary, $X \times \partial X$, associated to the Legendre manifold $\widetilde{G}(\lambda)$ given by Proposition 4 . It should be borne in mind that the dimension of this Legendre manifold is $2 n-1$ as this affects the formulæ when results of previous sections are quoted.

Recall that the scattering metric fixes the defining function of $\partial X, x$, up to an additive term in $x^{2} \mathcal{C}^{\infty}(X)$. This provides a trivilization of the line bundle $\left|N^{*} \partial X\right|^{a}$ which will play a rôle in the symbolic computations below. Let us in fact fix the defining function $x$ and a product decomposition of $X$ near $\partial X$. The choice of boundary defining function determines an operator

$$
\begin{gathered}
\Delta_{X} \in \operatorname{Diff}_{\mathrm{sc}}^{2}\left(X \times \partial X,{ }^{\mathrm{sc}} \Omega^{\frac{1}{2}}(X \times \partial X)\right) \text { by } \\
\Delta_{X}\left(u\left|\frac{d x}{x^{2}}\right|^{\frac{1}{2}}\left|\frac{d y}{x^{n-1}}\right|^{\frac{1}{2}}\left|\frac{d y^{\prime}}{x^{n-1}}\right|^{\frac{1}{2}}\right)=\Delta\left(u\left(\bullet, y^{\prime}\right)\left|\frac{d x}{x^{2}}\right|^{\frac{1}{2}}\left|\frac{d y}{x^{n-1}}\right|^{\frac{1}{2}}\right)\left|\frac{d y^{\prime}}{x^{n-1}}\right|^{\frac{1}{2}},
\end{gathered}
$$

where $\left(x, y ; y^{\prime}\right) \in X \times \partial X$ and $\Delta \in \operatorname{Diff}_{\mathrm{sc}}^{2}\left(X,{ }^{\mathrm{sc}} \Omega^{\frac{1}{2}}(X)\right)$ is the Laplace operator acting on scattering half-densities as in Lemma 7. 
As discussed in Proposition $4, \widetilde{G}(\lambda)$ is the unique Legendre pair which is characteristic for $\Delta_{X}-\lambda^{2}$ and satisfies

$$
\Lambda(\widetilde{G}(\lambda), \lambda)=N^{*} \Delta .
$$

Thus, Proposition 17 applies and shows that for $K$ associated to $\widetilde{G}(\lambda)$ the operator with the kernel given by $Q_{\lambda}^{0}(K)$ is necessarily multiplication by a smooth function.

Proposition 18. For any $0 \neq \lambda \in \mathbb{R}$ there exists $K \in I_{s c}^{m, p}\left(X \times \partial X, \widetilde{G}(\lambda) ;{ }^{s c} \Omega^{\frac{1}{2}}\right)$ such that, with the notation of Proposition 16,

$$
\left(\Delta_{X}-\lambda^{2}\right) K \in \dot{\mathcal{C}}^{\infty}\left(X \times \partial X ;{ }^{s c} \Omega^{\frac{1}{2}}\right) \text { and } Q_{\lambda}^{0}(K)=\mathrm{Id} .
$$

Here, $m$ and $p \in \mathbb{R}$ are determined by (14.6) so that the order of the Legrendre distribution is 0 , namely

$$
p=-\frac{1}{4} \text { and } m=-\frac{2 n-1}{4} .
$$

As a preliminary step towards the construction of $K$ we compute the subprincipal symbol of the extended Laplacian $\Delta_{X}$.

Lemma 14. Let $p=p_{0}+x p_{1} \in \rho_{\sigma}^{-2} \mathcal{C}^{\infty}\left({ }^{s c} \bar{T}^{*}(X \times \partial X)\right)$ be the expansion, (10.6), of the symbol of $\Delta_{X}-\lambda^{2} \in \operatorname{Diff}_{s c}^{2}\left(X \times \partial X,{ }^{s c} \Omega^{\frac{1}{2}}(X \times \partial X)\right)$. Then the lift of $p_{1}$ from $G$ extends smoothly to a function on $\widehat{G}$ which satisfies

$$
\left.\beta^{*}\left(\left.p_{1}\right|_{G}\right)\right|_{\partial \widehat{G}}=-i(n-1) \beta^{*} \tau \text {. }
$$

Proof. Let $p^{\prime}$ denote the symbol of $\Delta$ as an element of $\Psi_{\mathrm{sc}}^{2,0}\left(X,{ }^{\mathrm{sc}} \Omega^{\frac{1}{2}}\right)$. By Lemma 7 , $p^{\prime}=\tau^{2}+h(x, y, \mu)$. At $\mu=0$, which is well defined for a fixed defining function $x, h$ vanishes quadratically and hence for any $y \in \partial X, p_{0} /\left.x\right|_{\{\mu=0\} \cap G_{y}(\lambda)}=0$. Thus the only contribution to (15.3) comes from the action of $\Delta_{X}$ on the additional halfdensity factor $\left|d y^{\prime} / x^{n-1}\right|^{\frac{1}{2}}$ in ${ }^{\operatorname{sc}} \Omega^{\frac{1}{2}}(X \times \partial X)$. Again, because the defining function $x$ is fixed, the projection

$$
\pi_{L}:{ }^{\mathrm{sc}} T^{*}(X \times \partial X) \rightarrow{ }^{\mathrm{sc}} T^{*} X
$$

is well defined and the symbol of $\Delta_{X}$ acting on ${ }^{\text {sc }} \Omega^{\frac{1}{2}}(X \times \partial X)$ is

$$
\pi_{L}^{*}\left(j\left(x^{-(n-1) / 2} \Delta x^{(n-1) / 2}\right)\right) .
$$

At $\mu=0$ the new contribution comes from the $\left(x^{2} D_{x}\right)^{2}$ term in $\Delta$ and it is equal to $\pi_{L}^{*}\left(j\left(-i x(n-1) x^{2} D_{x}+\mathcal{O}\left(x^{2}\right)\right)\right)$. Thus the symbol of $\Delta_{X}$ at $\mu=0$ is

$$
\pi_{L}^{*} p_{0}-i x(n-1) \tau
$$

which gives (15.3).

Proof of Proposition 18. We now proceed to the construction of the parametrix for the Poisson kernel. First we seek a Legendre distribution $K^{b} \in I_{\mathrm{sc}}^{-\frac{2 n-1}{4},-\frac{1}{4}}(X \times$ $\left.\partial X, \widetilde{G}(\lambda) ;{ }^{\mathrm{sc}} \Omega^{\frac{1}{2}}\right)$ satisfying

$$
\begin{gathered}
\left(\Delta_{X}-\lambda^{2}\right) K^{\mathrm{b}}=I_{\mathrm{sc}}^{\frac{3}{4}}\left(X \times \partial X, G^{\sharp}(-\lambda) ;{ }^{\mathrm{sc}} \Omega^{\frac{1}{2}}\right) \text { and } \\
Q_{\lambda}^{0}\left(K^{\mathrm{b}}\right)=\mathrm{Id} .
\end{gathered}
$$


We shall construct $K^{b}$ as an asymptotic sum

$$
K^{b} \sim \sum_{j=0}^{\infty} K_{j}, K_{j} \in I_{\mathrm{sc}}^{-\frac{2 n-1}{4}+j,-\frac{1}{4}}\left(X \times \partial X, \widetilde{G}(\lambda) ;{ }^{\mathrm{sc}} \Omega^{\frac{1}{2}}\right)
$$

in the sense of Lemma 10 . Thus $K_{0}$ should satisfy

$$
\begin{gathered}
\left(\Delta_{X}-\lambda^{2}\right) K_{0} \in I_{\mathrm{sc}}^{-\frac{2 n-1}{4}+2, \frac{3}{4}}\left(X \times \partial X, \widetilde{G}(\lambda) ;{ }^{\mathrm{sc}} \Omega^{\frac{1}{2}}\right) \\
\text { and } \sigma_{0}\left(Q_{\lambda}^{0}\left(K_{0}\right)\right)=\sigma_{0}(\mathrm{Id}),
\end{gathered}
$$

and then the higher order terms should successively satisfy

$$
\left(\Delta_{X}-\lambda^{2}\right) K_{j}+\left(\Delta_{X}-\lambda^{2}\right)\left(\sum_{l=0}^{j-1} K_{l}\right) \in I_{\mathrm{sc}}^{-\frac{2 n-1}{4}+j+2, \frac{3}{4}}\left(X \times \partial X, \widetilde{G}(\lambda) ;{ }^{\mathrm{sc}} \Omega^{\frac{1}{2}}\right) .
$$

Near $G \cap G^{\sharp}(\lambda)$, that is where $G$ is smooth and satisfies (13.13), we can construct $K_{j}$ as Legendrian distributions associated to the smooth $G$ and of order $j-(2 n-$ 1)/4. Lemma 11 and Proposition 17 show that the second condition in (15.6) alone guarantees the second condition in (15.4). In fact, it is convenient to construct the $K_{j}$ rather explicitly (sc-microlocally) near the 'initial surface' $G^{\sharp}(\lambda)$. The Legendre variety $\widetilde{G}$ is smooth and is a graph over $\partial X \times \partial X$ near the diagonal. Hence, as follows from Proposition 4 , it is parametrized by the function $\lambda \phi\left(y, y^{\prime}\right)$ where $\phi\left(y, y^{\prime}\right)=\cos d\left(y, y^{\prime}\right) y$ with $d\left(y, y^{\prime}\right)$ being the local distance in $\partial X$, with respect to the induced metric. Then, just as in $\S 1$, the kernels $K_{j}$ takes the form of oscillatory functions

$$
K_{j}=x^{j} e^{i \lambda \phi\left(y, y^{\prime}\right) / x} a_{j}\left(x, y, y^{\prime}, \lambda\right) \nu, a_{j} \in \mathcal{C}^{\infty}(X \times \partial X) .
$$

To see that (15.6) and (15.7) have (unique) solutions of this form we regard $y^{\prime}$ as a parameter and introduce Riemannian normal coordinates in $y$, centered at $y^{\prime}$ (with respect to the metric $h$ on $\partial X)$. Just as in Hadamard's parametrix construction the transport equation for $a_{j}^{\prime}=a_{j} \mid(x=0)$ takes the form

$$
\left(y \cdot \partial_{y}+j\right) a_{j}^{\prime}+b_{j} a_{j}^{\prime}=c_{j} \in \mathcal{C}^{\infty}(X \times \partial X)
$$

near $y=0$, where $c_{0} \equiv 0$ and $b_{j}$ vanishes quadratically at $y=0$. The equation for $a_{0}$ therefore has a unique smooth solution with $a_{0}^{\prime}(y, y) \in \mathcal{C}^{\infty}(\partial X)$ specified and the equations for the $a_{j}^{\prime}, j \geq 1$, have unique smooth solutions. Thus the $K_{j}$ exist sc-microlocally near $G^{\sharp}(\lambda)$.

To continue these $K_{j}$ globally we use the symbolic filtration in Proposition 14 . Thus we look for $K_{j}$ as in (15.5), satisfying (15.6) and (15.7) and restricting to the distributions just defined microlocally. The first of the condition in (15.6) and the condition (15.7) can be stated symbolically as

$$
\begin{gathered}
\hat{\sigma}_{\mathrm{sc},-\frac{2 n-1}{4}+1, \frac{3}{4}}\left(\left(\Delta_{X}-\lambda^{2}\right) K_{0}\right)=0 \\
\hat{\sigma}_{\mathrm{sc},-\frac{2 n-1}{4}+j+1, \frac{3}{4}}\left(\left(\Delta_{X}-\lambda^{2}\right) K_{j}\right)=-\hat{\sigma}_{\mathrm{sc},-\frac{2 n-1}{4}+j+1, \frac{3}{4}}\left(\left(\Delta_{X}-\lambda^{2}\right)\left(\sum_{l=0}^{j-1} K_{l}\right)\right) .
\end{gathered}
$$

By (13.11) in Proposition 15, these become first order equations along the integral curves of the rescaled Hamilton vector field on $\widehat{G}$. Away from the initial surface $G^{\sharp}(\lambda)$ (which is not part of $\widetilde{G}$ ) and $\partial \widehat{G}=\widehat{G}_{0}, V$ in (13.11) is a nonvanishing 
smooth vector field. Thus, by continuing the solutions to these equations provided by (15.8) near $G^{\sharp}(\lambda)$ we can construct solutions throughout the smooth part of $\widetilde{G}$. It only remains to discuss the solvability of these equations near $G^{\sharp}(-\lambda)$.

From (5.18) we see that, near $\widehat{G}_{0}, \widehat{G}$ can be parameterized by

$$
\left(y^{\prime}, S, \hat{\mu}\right) \mapsto\left(y, y^{\prime}, S, \hat{\mu}, \hat{\mu}^{\prime}\right), S=\left|\left(\mu, \mu^{\prime}\right)\right| .
$$

The computation of the rescaled Hamilton vector field (3.6) and (5.17) shows that on $\widehat{G}$ near $\widehat{G}_{0}=\{S=0\}$

$$
V=2 \lambda S \partial_{S}+S \widetilde{V}, \widetilde{V} \in \mathcal{V}_{\mathrm{b}}(\widehat{G}) .
$$

Using Lemma 14 it follows that

$$
\begin{aligned}
&\left(\frac{1}{2}+\left(-\frac{2 n-1}{4}+j\right)-\frac{2 n-1}{4}\right) \beta^{*}\left(x^{-1 \mathrm{sc}} H_{p}^{2,0} \mid x=0\right) \\
&+i\left(-\frac{1}{4}+\frac{2 n-1}{4}\right) S^{-1} V S+i \beta^{*} p_{1}=i S f, f \in \mathcal{C}^{\infty}(\widehat{G}) .
\end{aligned}
$$

The left hand side of (13.11) therefore becomes

$$
\frac{1}{i} S\left(\left(2 \lambda \partial_{S}+\widetilde{V}+f+g\right) \tilde{a}\right) \nu \otimes|d x|^{-\frac{2 n-1}{4}+1} \otimes|d S|^{\frac{n-1}{2}}, \tilde{a} \in \mathcal{C}^{\infty}(\widehat{G}),
$$

where $\nu$ is some trivializing section of $\Omega_{\mathrm{b}}^{\frac{1}{2}}$ near $\partial \widehat{G}$ and both $f$ and $g$ are in $\mathcal{C}^{\infty}(\widehat{G})$. The transport equations in (15.6) and (15.7) therefore have smooth solutions on the whole of $\widehat{G}$, up to the boundary $\widehat{G}_{0}$.

Summing the $K_{j}$ asymptotically this gives the desired solution $K^{\mathrm{b}}$ to (15.4). It remains to improve this to a parametrix $K$ as in (15.2). To do this we need to improve the remainder term in (15.4). In fact it is already a little better than indicated.

Lemma 15. If $K^{b} \in I_{s c}^{-\frac{1}{4}(2 n-1),-\frac{1}{4}}\left(X \times \partial X, \widetilde{G}(\lambda) ;^{s c} \Omega^{\frac{1}{2}}\right)$ satisfies (15.4) then

$$
\left(\Delta_{X}-\lambda^{2}\right) K^{b} \in I_{s c}^{\frac{7}{4}}\left(X \times \partial X, G^{\sharp}(\lambda) ;{ }^{s c} \Omega^{\frac{1}{2}}\right) .
$$

Proof. Set $H=\left(\Delta_{X}-\lambda^{2}\right) K^{b}$. If $f \in \mathcal{C}^{\infty}\left(\partial X \times \partial X, \Omega^{\frac{1}{2}}\right)$ then by assumption (15.4)

$$
B(H, f)=x^{(n+1) / 2} Q_{-\lambda}(H, f)(x) e^{-i \lambda / x}
$$

where $Q_{-\lambda}(H, f) \in \mathcal{C}^{\infty}\left(X \times \partial X,{ }^{\text {sc }} \Omega^{\frac{1}{2}}\right)$. We need to show that $Q_{-\lambda}(H, f)(0)=0$ for all $f \in \mathcal{C}^{\infty}\left(\partial X \times \partial X, \Omega^{\frac{1}{2}}\right)$ since this is precisely (15.13).

Now, recall (3.12) of [10] (see also the proof of Proposition 12 there)

$$
\Delta=x \tilde{I}(Q) x+G, \tilde{I}(Q)=\left(x D_{x}\right)^{2}+i n x D_{x}-(n-1)+\widetilde{\Delta}_{0} .
$$

Here $G \in x^{2} \operatorname{Diff}_{\mathrm{c}}^{2}(X) \cap x \operatorname{Diff}_{\mathrm{sc}}^{2}(X)$, and $\widetilde{\Delta}_{0}$ is an extension of the Laplacian on $\partial X$ to $X$; $\operatorname{Diff}_{\mathrm{sc}}^{2}(X)$ is the set of scattering differential operators (generated by $x^{2} D_{x}$ and $x D_{y_{j}}$ ) and Diff ${ }_{\mathrm{c}}^{2}(X)$ is the set of cusp operators (generated by $x^{2} D_{x}$ and $D_{y_{j}}$ ). Then writing $B\left(K^{\mathrm{b}}, f\right)=B_{0}\left(K^{\mathrm{b}}, f\right)\left|d x / x^{2}\right|^{\frac{1}{2}}$,

$$
\begin{aligned}
B_{0}(H, f)=\left(\left(x^{2} D_{x}\right)^{2}+i x(n-1) x^{2} D_{x}-\lambda^{2}\right) & B_{0}\left(K^{b}, f\right) \\
+ & x^{2} B\left(K^{b}, \widetilde{\Delta}_{0} f\right)+x^{2} B_{0}\left(G_{0} K^{b}, f\right)
\end{aligned}
$$


where $G=x^{2} G_{0}$ with $G_{0} \in \operatorname{Diff}_{\mathrm{c}}^{2}(X)$. From Proposition 16 it follows that

$$
\begin{aligned}
B_{0}\left(K^{b}, f\right), B_{0}\left(K^{b}, \widetilde{\Delta}_{0} f\right), B_{0}\left(G_{0}, f\right) \in \\
x^{(n-1) / 2} e^{i \lambda / x} \mathcal{C}^{\infty}([0, \epsilon))+x^{(n-1) / 2} e^{-i \lambda / x} \mathcal{C}^{\infty}([0, \epsilon)) .
\end{aligned}
$$

Since

$$
\begin{aligned}
& \left(\left(x^{2} D_{x}\right)^{2}+i x(n-1) x^{2} D_{x}-\lambda^{2}\right) x^{p} \exp ( \pm i \lambda / x)= \\
& \left( \pm i \lambda(2 p-n+1) x^{p+1}+p^{2} x^{p+2}\right) \exp ( \pm i \lambda / x)
\end{aligned}
$$

we conclude that

$$
B_{0}(H, f) \in x^{(n-1) / 2+2} e^{i \lambda / x} \mathcal{C}^{\infty}([0, \epsilon))+x^{(n-1) / 2+2} e^{-i \lambda / x} \mathcal{C}^{\infty}([0, \epsilon)),
$$

giving the desired result.

To remove the improved error we will use:

Lemma 16. For $0 \neq \lambda \in \mathbb{R}$ and $b \in \mathcal{C}^{\infty}\left(X \times \partial X,{ }^{s c} \Omega^{\frac{1}{2}}\right)$ there exists $a \in \mathcal{C}^{\infty}(X \times$ $\left.\partial X,{ }^{s c} \Omega^{\frac{1}{2}}\right)$ such that $U=x^{(n-1) / 2} e^{ \pm i \lambda / x}$ a satisfies

$$
\left(\Delta_{X}-\lambda^{2}\right) U-x^{(n+3) / 2} e^{ \pm i \lambda / x} b \in \dot{\mathcal{C}}^{\infty}\left(X \times \partial X,{ }^{s c} \Omega^{\frac{1}{2}}\right) .
$$

Proof. This is essential Proposition 12 of [10]. The proof proceeds by iteration based on the following consequence of (15.15) and (15.17)

$$
\left(\Delta-\lambda^{2}\right) x^{p} \exp (-i \lambda / x) b=x^{p+1} \exp (-i \lambda / x) g, g=-i \lambda(2 p-n+1) b+O(x) .
$$

This allows the Taylor series of $a$ to be constructed. Summing this series using Borel's lemma gives $U$.

Applying Lemma 16 to the error term in (15.13) allows the kernel $K^{\text {b }}$ to be corrected to give a parametrix $K$ in the sense of (15.2) and hence completes the proof of Proposition 18.

\section{Proof of main theorem}

The action on half-densities, which simplifies the symbolic properties, can now be dropped and the results of the previous sections give

Proposition 19. For any $0 \neq \lambda \in \mathbb{R}$ and any scattering metric on a compact manifold with boundary the Poisson operator $P(\lambda)$, defined by (0.4), has Schwartz kernel given by a scattering Legendre distribution

$$
P(\lambda) \in I_{s c}^{-\frac{2 n-1}{4},-\frac{1}{4}}(X \times \partial X, \widetilde{G}(\lambda)) .
$$

Proof. Starting from the parametrix $K$ given by Proposition 18 we first remove the error term in (15.2). To do so we use Proposition 14 of [10] which shows that, given $F \in \dot{\mathcal{C}}^{\infty}(X \times \partial X)$ there exists $U^{\prime} \in x^{(n-1) / 2} e^{ \pm i \lambda / x} \mathcal{C}^{\infty}(X \times \partial X)$ such that $\left(\Delta_{X}-\lambda^{2}\right) U^{\prime}=F^{\prime}$. This actually involves a slight extension of the construction there in which the data, and hence the solution, depend smoothly on parameters but this follows as usual from the uniqueness of the solution.

Thus it only remains to show that a kernel $K \in I_{\mathrm{sc}}^{m, p}\left(X \times \partial X, \widetilde{G}(\lambda) ;{ }^{\mathrm{sc}} \Omega^{\frac{1}{2}}\right)$ satifying the exact form of (15.2)

$$
\left(\Delta_{X}-\lambda^{2}\right) K=0 \text { and } Q_{\lambda}^{0}(K)=\mathrm{Id}
$$


is actually the kernel of the Poisson operator defined by (0.4). Thus it is only necessary to check that for $g \in \mathcal{C}^{\infty}\left(\partial X, \Omega^{\frac{1}{2}}\right)$

$$
x^{\frac{n-1}{2}} \int_{\partial X} K\left(\bullet, y^{\prime}\right) g\left(y^{\prime}\right)=x^{\frac{n-1}{2}}\left(e^{i \frac{\lambda}{x}} f^{\prime}+e^{-i \frac{\lambda}{x}} f^{\prime \prime}\right),
$$

where $f^{\prime}, f^{\prime \prime} \in \mathcal{C}^{\infty}\left(X,{ }^{\mathrm{sc}} \Omega^{\frac{1}{2}}\right)$. Formally, Proposition 16 gives (16.3) with $f^{\prime}=$ $Q_{+}\left(K, \delta_{\bullet} \otimes g\right)$ and $f^{\prime \prime}=Q_{-}\left(K, \delta_{\bullet} \otimes g\right)$ where for $y \in \partial X, \delta_{y} \in \mathcal{C}^{\infty}\left(\partial X_{y}, \Omega^{\frac{1}{2}} \otimes\right.$ $\left.\mathcal{C}^{-\infty}\left(\partial X, \Omega^{\frac{1}{2}}\right)\right)$ is the pullback of the kernel of the identity on $\mathcal{C}^{\infty}\left(\partial X,{ }^{\text {sc }} \Omega^{\frac{1}{2}}\right)$ by $y^{\prime} \mapsto\left(y, y^{\prime}\right)$. On the other hand, by Lemma $13,\left.(d / d x)^{l} Q_{ \pm}(K, \delta \bullet \otimes g)\right|_{x=0}$ are given by the push-forward of Lagrangian distributions with the relations given by Proposition 4 . The standard calculus of wave front sets for push-forwards (see Theorem 8.2 .12 in [7]) then shows that $Q_{ \pm}(K, \delta \bullet \otimes g) \in \mathcal{C}^{\infty}\left(\partial X, \Omega^{\frac{1}{2}}\right)$. Hence, approximating $\delta_{y}$ by a family $\phi_{y}^{\epsilon} \in \mathcal{C}^{\infty}\left(\partial X_{y}, \Omega^{\frac{1}{2}} \otimes \mathcal{C}^{\infty}\left(\partial X, \Omega^{\frac{1}{2}}\right)\right)$ gives the desired smoothness of $f_{ \pm}$.

The main theorem as stated in the introduction is now a consequence of Propositions 16 and 4, which together show that the scattering matrix, which is given by the boundary value in the sense of (0.3), has as kernel aLagrangian distribution associated to geodesic flow at time $\pi$ on the boundary; from (14.6) it is of order 0 .

The smooth dependence of the scattering operator, as a function of $0 \neq \lambda \in \mathbb{R}$ follows from these constructions. The canonical relation with which it is associated is independent of $\lambda$. The approximate $\mathbb{R}^{+}$invariance of the problem shows that

Lemma 17. The scattering matrix of a scattering metric is such that $\partial_{\lambda} A(\lambda)$ is of order -1 , i.e. the symbol of $A(\lambda)$ is (locally) independent of $\lambda$.

Even more than this can be seen in the 'product case' in which the metric takes the form

$$
g=\frac{d x^{2}}{x^{4}}+\frac{h}{x^{2}}
$$

in some product decomposition near the boundary with $h$ a fixed metric on the boundary, i.e. independent of $x$. Then $\partial_{\lambda} A(\lambda)$ is a smoothing operator. In this case the scattering phase can be defined as having derivative

$$
\frac{d s(\lambda)}{d \lambda}=\operatorname{tr}\left(A(\lambda)^{-1} \frac{d A(\lambda)}{d \lambda}\right)
$$

As in the perturbed Euclidean case, see [11], [12], it is to be expected that this function should be the Fourier transform of the appropriately regularized trace of the wave group.

\section{REFERENCES}

[1] S. Agmon and L. Hörmander, Asymptotic properties of solutions of differential equations with simple characteristics, J. Analyse Math. 30 (1976), 1-38.

[2] J. Brüning and V.W. Guillemin (Editors), Fourier integral operators, SpringerVerlag, Berlin Heidelberg New York Tokyo, 1994.

[3] J.J. Duistermaat and L. Hörmander, Fourier integral operators, II, Acta Math. 128 (1972), 183-269.

[4] V.W. Guillemin and S. Sternberg, Geometrical asymptotics, AMS Surverys, vol. 14, AMS, Providence, R.I., 1977. 
[5] L. Hörmander, Fourier integral operators, I, Acta Math. 127 (1971), 79-183, See also [2].

[6] The Weyl calculus of pseudo-differential operators, Comm. Pure Appl. Math. 32 (1979), 359-443.

[7] - The analysis of linear partial differential operators, Grundlehren Math. Wiss. 274 and 275, vol. 3 and 4, Springer-Verlag, Berlin Heidelberg NewYork Tokyo, 1986.

[8] P.D. Lax and R.S. Phillips, Decaying modes for the wave equation in the exterior of an obstacle, Comm. Pure Appl. Math. 22 (1969), 737-787.

[9] R.B. Melrose, Pseudodifferential operators, corners and singular limits, Proc. Int. Congress of Mathematicians, Kyoto, Springer Verlag, 1990, pp. 217-234.

[10] _ Spectral and scattering theory for the laplacian on asymptotically Euclidian spaces, Spectral and scattering theory (M. Ikawa, ed.), Marcel Dekker, 1994.

[11] _ Geometric scattering theory, Cambridge Universtiy Press, 1995, To appear.

[12] D. Robert, Relative time-delay for perturbations of elliptic operators and semiclassical asymptotics, To Appear.

[13] _ Relative time delay and trace formula for long range perturbation of Laplace's operator, Operator theory; Advances and Applications, vol. 57, Birkäuser Verlag, Basel, 1992.

Department of MAThematics, MiT

E-mail address: rbm@@math.mit.edu

Department of Mathematics, The Johns Hopkins University

E-mail address: mz@@math.jhu.edu 